\title{
Big Bang Observer and the neutron-star-binary subtraction problem
}

\author{
Curt Cutler \\ Jet Propulsion Laboratory, California Institute of Technology, Pasadena, California 91109, USA \\ Jan Harms \\ Max-Planck-Institut für Gravitationsphysik and Universität Hannover, Callinstraße 38, 30167 Hannover, Germany
}

(Received 16 November 2005; published 8 February 2006)

\begin{abstract}
The Big Bang Observer (BBO) is a proposed space-based gravitational-wave $(\mathrm{GW})$ mission designed primarily to search for an inflation-generated GW background in the frequency range $\sim 10^{-1} \mathrm{~Hz}-1 \mathrm{~Hz}$. The major astrophysical foreground in this range is gravitational radiation from inspiralling compact binaries. This foreground is expected to be much larger than the inflation-generated background, so to accomplish its main goal, BBO must be sensitive enough to identify and subtract out practically all such binaries in the observable universe. It is somewhat subtle to decide whether BBO's current baseline design is sufficiently sensitive for this task, since, at least initially, the dominant noise source impeding identification of any one binary is confusion noise from all the others (rather than instrumental noise). Here we present a self-consistent scheme for deciding whether BBO's baseline design is indeed adequate for subtracting out the binary foreground. We conclude that the current baseline should be sufficient. However, if BBO's sensitivity were degraded by a factor 2 from the current baseline, then its ability to detect an underlying primordial background would depend critically on the value of $\rho_{\text {th }}$, the threshold signal-to-noise ratio marking the boundary between detectable and undetectable sources. If BBO's sensitivity were degraded by a factor 4 from the current baseline, it could not detect a primordial background below $\Omega_{\mathrm{GW}} \sim 10^{-15}$. It is impossible to perfectly subtract out each of the binary inspiral waveforms, so an important question is how to deal with the "residual" errors in the post-subtraction data stream. We sketch a strategy of "projecting out" these residual errors, at the cost of some effective bandwidth. We also provide estimates of the sizes of various post-Newtonian effects in the inspiral waveforms that must be accounted for in the BBO analysis.
\end{abstract}

DOI: 10.1103/PhysRevD.73.042001

\section{INTRODUCTION}

The Big Bang Observer (BBO) is a proposed spacebased gravitational wave (GW) mission designed to search for stochastic gravitational-wave background generated in the very early universe $[1,2]$. The design goal is to be able to detect primordial GWs with energy density $\Omega_{\mathrm{GW}}(f) \gtrsim$ $10^{-17}$ in the frequency band $10^{-1} \mathrm{~Hz}<f<1 \mathrm{~Hz}$. Standard, slow-roll inflation predicts $\Omega_{\mathrm{GW}}(f) \lesssim 10^{-16}-$ $10^{-15}[3]$.

To achieve this sensitivity to a primordial GW background, it will first be necessary to subtract from the BBO data stream the GW foregv vround generated by $\sim 10^{5}-10^{6}$ neutron star-neutron star (NS-NS), neutron star-black hole (NS-BH), and black hole-black hole (BH-BH) binary mergers, out to $z \sim 5$. This foreground "noise" has an amplitude substantially greater than BBO's instrumental noise, which in turn is probably substantially greater than the amplitude of the sought-for primordial GWs. To achieve BBO's goal, the GWs from the merger foreground must be subtracted to a level well below that of the primordial background. This means that the amplitude of the residual, post-subtraction foreground must be $\lesssim 10^{-2.5}$ of the presubtraction level.

Will it be possible for BBO data analysts to subtract out the binary merger foreground to this accuracy? This question is nontrivial to answer precisely because confusion
PACS numbers: $04.80 . \mathrm{Nn}, 04.25 . \mathrm{Nx}, 04.30 . \mathrm{Db}, 95.75 . \mathrm{Wx}$

noise from unresolved mergers can in principle dominate the BBO noise spectrum. To decide which mergers are unresolvable, one needs to know the full BBO noise curve, including the level of confusion noise from the unresolvable mergers. But to determine the level of confusion noise, of course one needs to know which mergers are unresolvable. Clearly, one needs somehow to solve both these problems simultaneously.

The focus of our investigations will be on NS-NS mergers, since these are the most problematic for BBO. The less numerous $\mathrm{BH}-\mathrm{BH}$ and $\mathrm{BH}-\mathrm{NS}$ merger events will have higher signal-to-noise ratios and therefore should be easier to subtract. If we find the NS-NS mergers can be almost fully subtracted from the BBO data stream, then the same should be true for the BH-BH and BH-NS mergers.

How, in practice, will almost all the NS-NS mergers be subtracted out? We imagine that something like the following iterative scheme could be used: begin by resolving and subtracting out the brightest merging binaries (i.e., those with highest signal-to-noise-ratio), then resolve and subtract the next brightest ones, etc. - regularly updating all the parameters of the subtracted binaries, as one goes along, to give the best global fit. Each subtraction decreases the foreground confusion noise and so increases the distance out to which NS binaries can be resolved. Will such a scheme suffice for BBO? The aim of this paper is to answer that question without actually having to carry out 
the whole procedure. We develop a method for determining the likely efficacy of foreground subtraction in a selfconsistent manner. Our method is (very roughly) as follows. Imagine that BBO is surrounded by a huge sphere out to some redshift $\bar{z}$, such that NS-NS mergers inside the sphere (i.e., at redshifts less than $\bar{z}$ ) can all be individually resolved and subtracted (using realistic computational power), while none of the sources outside the sphere is resolvable. This redshift $\bar{z}$ marking the boundary of the resolvable sources is not known initially, so we start with a reasonable guess. We then calculate the confusion noise due to all NS-NS mergers (NSm) at redshifts greater than $\bar{z}$, $S_{\mathrm{h}}^{\mathrm{NSm},>\bar{z}}(f)$, which we add to the instrumental noise $S_{\mathrm{h}}^{\text {inst }}(f)$ to obtain the total noise:

$$
S_{\mathrm{h}}^{\text {tot }}(f)=S_{\mathrm{h}}^{\text {inst }}(f)+S_{\mathrm{h}}^{\mathrm{NSm},>\bar{z}}(f) .
$$

One can use this total noise level, $S_{\mathrm{h}}^{\text {tot }}(f)$, to improve one's estimate of $\bar{z}$, and iterate this procedure until $\bar{z}$ converges.

Actually, of course, the detectability of any particular NS-NS binary depends not just on its distance (or redshift), but also on $\mu \equiv \hat{L} \cdot \hat{N}$, where $\hat{L}$ is the normal to the binary's orbital plane and $\hat{N}$ points along our line-of-sight. (The binary's detectability also depends, of course, on the other three angles describing the binary's orientation and position on the sky, but to a much lesser extent.) Our calculation does properly account for the $\mu$-dependence of the binary's detectability; i.e., we take $\bar{z}$ to be a function of $\mu$, not a single number.

We stress that there are actually two different sorts of confusion noise associated with merging binaries: the full signals from unresolved binaries (mentioned above), and the small errors that inevitably occur when waveforms from resolved mergers are subtracted out of the data. In Sec. IV we propose a method for dealing with these residual errors, by projecting out the subspace in which these errors can lie, at the cost of some bandwidth. We also estimate that this fractional decrease in BBO's bandwidth is small enough that for our purpose (deciding whether an iterative subtraction scheme is feasible) it can be neglected.

We remark that our calculation is quite similar in spirit to a recent analysis of WD-binary subtraction in LISA data analysis, by Cornish et al. [4], which appeared when our own work was already at an advanced stage. In both cases, the idea is to use the requirement of self-consistency to arrive at a unique estimate of the efficacy of foreground subtraction, without actually coding up the whole analysis pipeline and testing it on simulated data.

We also remark that a recent paper by Buonanno et al. [5] estimates that supernova explosions could provide another important BBO foreground, via the GW memory effect, but only if the anisotropy of neutrino emission is quite high, on average. For the rest of this paper we will neglect the possibility of a large foreground from supernovae.
The organization of the rest of this paper is as follows. In Sec. II we give a brief overview of the BBO mission, its design sensitivity, and the foreground produced by merging NS binaries. In Sec. III we briefly explain why the most distant NS-NS binaries are effectively a noise source when it comes to resolving more nearby ones. In Sec. IV we summarize our proposed strategy of dealing with any residual subtraction errors by projecting them out. In Sec. V we provide estimates regarding the importance of eccentricity, NS spin, and high-order post-Newtonian (PN) effects in correctly subtracting out the resolved mergers. Besides being important for any future implementation of a BBO analysis pipeline, this catalog of effects is useful in estimating the threshold signal-to-noise ratio (SNR) $\rho_{\text {th }}$ required to detect NS-NS mergers. In Sec. VI we take a first cut at estimating $\rho_{\text {th }}$, which we assume will be set by the then-available computational power. Our equations for self-consistently determining the efficacy of foreground subtraction are developed in Sec. VII. We solve these equations for a variety of assumptions regarding the NS merger rate, the detection threshold $\rho_{\text {th }}$, and BBO's instrumental noise level, and display the solutions in Sec. VIII. We summarize our conclusions in Sec. IX. The derivation of one of the equations in Sec. VII is relegated to Appendix A.

We use units in which $G=c=1$. Therefore, everything can be measured in the fundamental unit of seconds. However, for the sake of familiarity, we also sometimes express quantities in terms of $\mathrm{yr}, \mathrm{Mpc}$, or $M_{\odot}$, which are related to our fundamental unit by $1 \mathrm{yr}=3.1556 \times 10^{7} \mathrm{~s}$, $1 \mathrm{Mpc}=1.029 \times 10^{14} \mathrm{~s}$, and $1 M_{\odot}=4.926 \times 10^{-6} \mathrm{~s}$.

For concreteness, we assume the universe corresponds to a flat Friedmann-Robertson-Walker model, with the universe's matter and vacuum energy densities being given by $\Omega_{\mathrm{m}}=0.33$ and $\Omega_{\Lambda}=0.67$, respectively. Our fiducial value for the Hubble constant is $H_{0}=70 \mathrm{kms}^{-1} \mathrm{Mpc}^{-1}$.

\section{OVERVIEW OF BBO AND THE NS-BINARY BACKGROUND}

\section{A. BBO}

BBO is essentially a follow-on mission to LISA, the planned Laser Interferometer Space Antenna [6], but optimized to detect GWs generated by parametric amplification during inflation. (For a review of inflation-generated GWs, see Allen [7] and references therein). In the LISA band, $10^{-5} \mathrm{~Hz}-10^{-1} \mathrm{~Hz}$, an inflation-generated signal with $\Omega_{\mathrm{GW}} \lesssim 10^{-15}$ would be completely covered up by the foreground produced by galactic and extra-galactic white-dwarf binaries. By contrast, $\mathrm{BBO}$ will have its best sensitivity in the range $\sim 0.1 \mathrm{~Hz}-1 \mathrm{~Hz}$. This band avoids the GW foreground produced by all the white-dwarf binaries in the universe, which cuts off at $f \leqslant 0.2 \mathrm{~Hz}$ (where the most massive of the WD binaries merge). In the BBO band, the dominant foreground GW sources are inspiralling NS-NS, NS-BH, and BH-BH binaries. BBO's baseline 
design, and corresponding instrumental noise curve, have been set in large part by the requirement that one must be able to individually identify practically all such inspiral signals and subtract them out of the data. An initial rough estimate suggested that the baseline "specs" in Table I are adequate for this purpose [1]; our primary task in this paper is to examine that issue much more carefully.

The current BBO design calls for four constellations of three satellites each, all following heliocentric orbits at a distance of 1 AU from the Sun (see Fig. 1). Each 3-satellite constellation can be thought of as a "short-armed LISA". Two of the constellations overlap to form a "Jewish star"; the other two are ahead and behind by $2 \pi / 3$ radians, respectively. Briefly, the idea behind this orbital geometry is that $\Omega_{\mathrm{GW}}(f)$ will be measured by cross-correlating the outputs of the two overlapping constellations in the Jewish star (much as LIGO attempts to measure $\Omega_{\mathrm{GW}}(f)$ by crosscorrelating the outputs of the Livingston and Hanford interferometers [8]). The other two constellations give BBO its angular resolution: $\Delta \theta \sim 10^{-2}(\mathrm{SNR})^{-1}$ radians. It is not clear whether this angular resolution is strictly necessary for the purpose of measuring $\Omega_{\mathrm{GW}}(f)$, but it will be immensely useful for BBO's secondary goal - to identify, map, and accurately determine the physical parameters of practically all merging compact binaries in the observable universe.

From the output of each 3-satellite constellation (i.e., each "mini-LISA"), using time-delay interferometry (TDI) one can synthesize data streams that are free of laser phase noise and optical bench noise [9-11]. A particularly convenient set of TDI variables to work with is $\{A, E, T\}$; all the GW information registered by each mini-LISA is encoded in these variables, plus the noises in these 3 channels are uncorrelated with each other (i.e., they are statistically independent). Then, for instance, it is straightforward to find, for any source, the particular combination of $\{A, E, T\}$ that yields the optimum detection statistic, and so to determine LISA's optimum sensitivity to that source [10].

For our purposes, however, the following simplified treatment is adequate. As is clear from Fig. 4 of Prince et al. [10], for NS-NS inspirals, each mini-LISA's sensitivity (using the optimum combination of the $A, E$, and $T$ channels) is practically equivalent to the sensitivity of two synthetic Michelson detectors, represented by the TDI

TABLE I. BBO parameters.

\begin{tabular}{lcc}
\hline \hline & Symbol & Value \\
\hline Laser power & $P$ & $300 \mathrm{~W}$ \\
Mirror diameter & $D$ & $3.5 \mathrm{~m}$ \\
Optical efficiency & $\epsilon$ & 0.3 \\
Arm length & $L$ & $5 \cdot 10^{7} \mathrm{~m}$ \\
Wavelength of laser light & $\lambda$ & $0.5 \mu \mathrm{m}$ \\
Acceleration noise & $\sqrt{S_{\text {acc }}}$ & $3 \cdot 10^{-17} \mathrm{~m} /\left(\mathrm{s}^{2} \sqrt{\mathrm{Hz}}\right)$ \\
\hline \hline
\end{tabular}

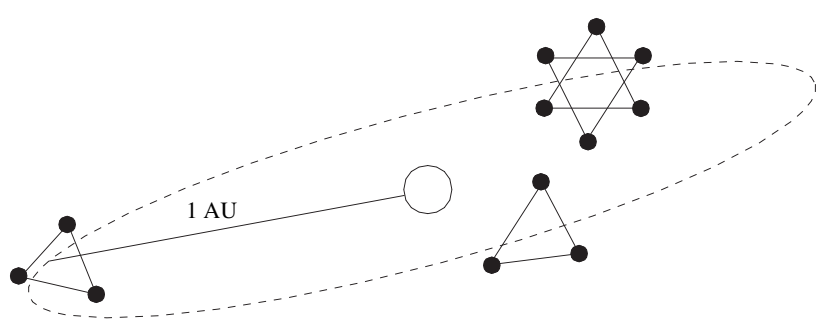

FIG. 1. The big bang observer (BBO) consists of four LISAlike triangular constellations orbiting the Sun at $1 \mathrm{AU}$. The GW background is measured by cross-correlating the outputs of the two overlapping constellations.

variables $X$ and $Y$. For our purposes, then, we can regard $\mathrm{BBO}$, which is made up of 4 mini-LISAs, as formally equivalent to 8 synthetic Michelson interferometers.

To construct the instrumental noise curve, $S_{\mathrm{h}}^{\text {inst }}(f)$, of each of these synthetic Michelson's, we used Larson's online "Sensitivity curve generator" [12], plugging in the parameters appropriate to $\mathrm{BBO}$, which are listed here in Table I.

The parameters we adopt as reference values here are taken from the BBO proposal [1]; these parameters do not necessarily represent the latest thoughts on the mission's design (which is a moving target), but do provide a convenient baseline for comparison. (Reference [1] also lists parameters for less and more ambitious versions of the BBO mission, referred to as "BBO-lite" and "BBOgrand," respectively, but in this paper we concentrate on the intermediate version, or "standard BBO".) In using the

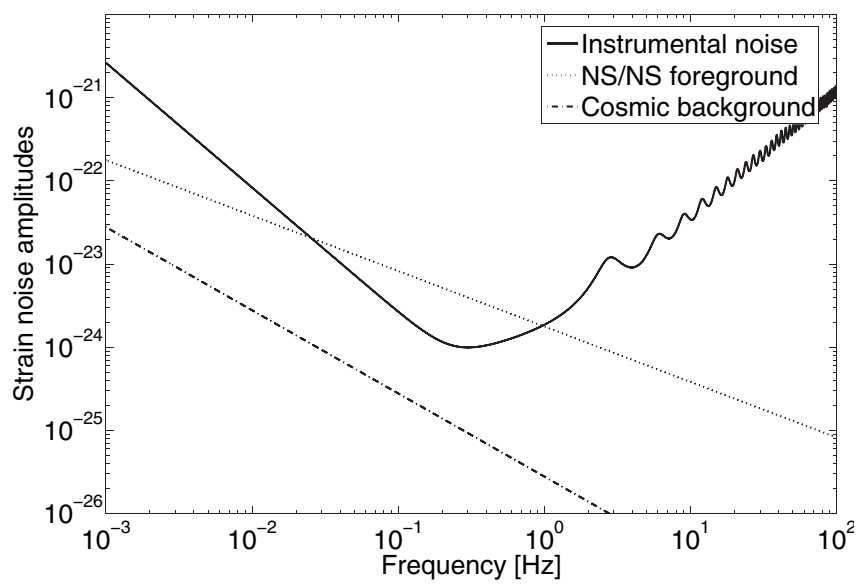

FIG. 2. Shows the amplitude of the instrumental noise, $\sqrt{f S_{\mathrm{h}}^{\text {inst }}(f)}$, compared to the amplitude of the (presubtraction) NS-binary foreground (plotted for $\dot{n}_{0}=10^{-7} \mathrm{Mpc}^{-3} \mathrm{yr}^{-1}$ ) and the sought-for cosmic $\mathrm{GW}$ background (plotted for $\Omega_{\mathrm{GW}}(f)=$ $10^{-16}$ ). Clearly, to reveal a cosmic GW background at this level, the NS foreground must be subtracted off, with fractional residual of $\lesssim 10^{-2.5}$. 
online generator, we have specified that the high-frequency part of $S_{\mathrm{h}}^{\text {inst }}$ is 4 times larger than the contribution from photon shot noise alone. This is the same choice made in Fig. 1 of the BBO proposal [1], and is consistent with assumptions typically made in drawing the LISA noise curve. As is conventional in the LISA literature, we take $S_{\mathrm{h}}(f)$ to be the single-sided, sky-averaged noise spectrum for each synthetic Michelson. This BBO instrumental noise curve is shown in Fig. 2.

\section{B. The NS-NS merger rate and the associated foreground noise level}

In this subsection we estimate the magnitude of the GW foreground from all NS-NS mergers. We denote the NS-NS merger rate (per unit proper time, per unit comoving volume) at redshift $z$ by $\dot{n}(z)$. The presentday density $n_{0}$ of merger remnants is related to $\dot{n}(z)$ by [13]

$$
n_{0}=\int_{0}^{\infty} \mathrm{d} z \frac{\dot{n}(z)}{(1+z) H(z)},
$$

where

$$
H(z) \equiv H_{0} \sqrt{\Omega_{\mathrm{m}}(1+z)^{3}+\Omega_{\Lambda}} .
$$

As is conventional, we define $\Omega_{\mathrm{GW}}(f)$ to be the universe's fractional energy in GWs, per logarithmic frequency interval:

$$
\Omega_{\mathrm{GW}}(f) \equiv \frac{1}{\rho_{\mathrm{c}}} \frac{\mathrm{d} \rho_{\mathrm{GW}}(f)}{\mathrm{d}(\ln f)},
$$

where $\rho_{c}=3 H_{0}^{2} /(8 \pi)$ is the universe's current energy density. Then the GW energy density (in the BBO band) due to (the inspiral phase of) all NS-NS mergers is given by [13]

$$
\begin{aligned}
\Omega_{\mathrm{GW}}^{\mathrm{NSm}}(f)= & \frac{8 \pi^{5 / 3}}{9} \frac{1}{H_{0}^{2}} \mathcal{M}^{5 / 3} f^{2 / 3} n_{0}\left\langle(1+z)^{-1 / 3}\right\rangle \\
= & 1.7 \times 10^{-12} h_{70}^{2}\left(\frac{\mathcal{M}}{1.22 M_{\odot}}\right)^{5 / 3}\left(\frac{f}{1 \mathrm{~Hz}}\right)^{2 / 3} \\
& \cdot\left(\frac{n_{0}}{10^{3} \mathrm{Mpc}^{-3}}\right)\left(\frac{\left\langle(1+z)^{-1 / 3}\right\rangle}{0.80}\right) .
\end{aligned}
$$

The term $\left\langle(1+z)^{-1 / 3}\right\rangle$ in Eq. (5) is the merger-rateweighted average of $(1+z)^{-1 / 3}$, given by

$$
\left\langle(1+z)^{-1 / 3}\right\rangle \equiv \frac{1}{n_{0}} \int_{0}^{\infty} \mathrm{d} z \frac{\dot{n}(z)}{(1+z)^{4 / 3} H(z)} .
$$

What is the universe's NS-NS merger rate history, $\dot{n}(z)$ ? It is convenient to regard $\dot{n}(z)$ as the product of two factors:

$$
\dot{n}(z)=\dot{n}_{0} \cdot r(z),
$$

where $\dot{n}_{0}$ is the merger rate today and $r(z)$ encapsulates the rate's time-evolution.
For $r(z)$, we adopt the following piecewise linear fit to the rate evolution derived in [14]:

$$
r(z)= \begin{cases}1+2 z & z \leq 1, \\ \frac{3}{4}(5-z) & 1 \leq z \leq 5, \\ 0 & z \geq 5\end{cases}
$$

For this $r(z)$ and our fiducial cosmological model, one has

$$
n_{0}=\dot{n}_{0} \cdot\left(2.3 \cdot 10^{10} \mathrm{yr}\right) \text {, }
$$

and $\left\langle(1+z)^{-1 / 3}\right\rangle=0.82$. (We note that, as stressed in [13], the value of $\left\langle(1+z)^{-1 / 3}\right\rangle$ is actually quite insensitive to one's choice of the function $r(z)$, generally being in the range $\sim 0.7-0.9$.)

The current NS-NS merger rate, $\dot{n}_{0}$, is also usefully regarded as the product of two factors: the current merger rate in the Milky Way and a factor that extrapolates from the Milky Way rate to the average rate in the universe. The NS-NS merger rate in the Milky Way has been estimated by several authors; it is still highly uncertain, but most estimates are in the range $10^{-6}-10^{-4} \mathrm{yr}^{-1}$ [15-17]. To extrapolate to the rest of the universe, Kalogera et al. [16] estimate that one should multiply the Milky Way rate by $1.1-1.6 \times 10^{-2} \cdot h_{70} \mathrm{Mpc}^{-3}$. That factor is obtained by extrapolating from the B-band luminosity density of the universe, and it is only a little larger than the extrapolation factor derived by Phinney in [18]. Given the large overall uncertainty, in this paper we will consider 3 possible rates: $\dot{n}_{0}=10^{-8}, 10^{-7}$, and $10^{-6} \mathrm{Mpc}^{-3} \mathrm{yr}^{-1}$.

How many NS-NS merger events $\Delta N_{\mathrm{m}}$ enter the BBO band during some observation time $\Delta \tau_{0}$ ? Summing the contributions from all redshifts, the rate $\dot{N} \equiv \Delta N_{\mathrm{m}} / \Delta \tau_{0}$ is easily shown to be

$$
\dot{N}=\int_{0}^{\infty} 4 \pi\left[a_{0} r_{1}(z)\right]^{2} \dot{n}(z) \frac{\mathrm{d} \tau_{1}}{\mathrm{~d} z} \mathrm{~d} z
$$

where (for our fiducial cosmology)

$$
\begin{gathered}
a_{0} r_{1}(z)=\frac{1}{H_{0}} \int_{0}^{z} \frac{\mathrm{d} z^{\prime}}{\sqrt{\left(1-\Omega_{\Lambda}\right)\left(1+z^{\prime}\right)^{3}+\Omega_{\Lambda}}}, \\
\frac{\mathrm{d} \tau_{1}}{\mathrm{~d} z}=\frac{1}{H_{0}} \frac{1}{1+z} \frac{1}{\sqrt{\left(1-\Omega_{\Lambda}\right)(1+z)^{3}+\Omega_{\Lambda}}} .
\end{gathered}
$$

This yields

$$
\Delta N_{\mathrm{m}}=3.0 \cdot 10^{5}\left(\frac{\Delta \tau_{0}}{3 \mathrm{yr}}\right)\left(\frac{\dot{n}_{0}}{10^{-7} \mathrm{Mpc}^{-3} \mathrm{yr}^{-1}}\right) .
$$

The time required for a NS-NS inspiral signal to sweep through the $\mathrm{BBO}$ band will typically be comparable to BBO's lifetime. More specifically, the time remaining until merger, from the moment the $\mathrm{GW}$ frequency sweeps through $f$, is given (to lowest post-Newtonian order) by

$$
t(f)=4.64 \times 10^{5} \mathrm{~s}\left(\frac{\mathcal{M}(1+z)}{1.22 M_{\odot}}\right)^{-5 / 3}\left(\frac{f}{1 \mathrm{~Hz}}\right)^{-8 / 3},
$$


where $\mathcal{M} \equiv \mu^{3 / 5} M^{2 / 5}$ is the so-called "chirp mass" of the binary. (Here $M$ is the binary's total mass and $\mu$ is its reduced mass.) Therefore, for two $1.4 M_{\odot}$ NSs, $f \approx$ $0.205 \mathrm{~Hz}, 0.136 \mathrm{~Hz}$, and $0.112 \mathrm{~Hz}$ at $1 \mathrm{yr}$, three years, and five years before merger, respectively.

Figure 3 plots the number of observable mergers during 3 years that occur closer than (any given) redshift $z$. We see that only $\sim 15 \%$ of mergers occur closer to us than $z=1$.

The (single-sided, sky-averaged) noise spectral density associated with any given GW background is [7]:

$$
S_{\mathrm{h}}^{\mathrm{GW}}=\frac{3}{2} \frac{H_{0}^{2}}{\pi^{2}} \frac{1}{f^{3}} \Omega_{\mathrm{GW}}(f)
$$

or

$$
\left[f S_{\mathrm{h}}^{\mathrm{GW}}(f)\right]^{1 / 2}=8.8 \times 10^{-25} \cdot h_{70}\left(\frac{\Omega_{\mathrm{GW}}(f)}{10^{-12}}\right)^{1 / 2}\left(\frac{1 \mathrm{~Hz}}{f}\right) .
$$

The effective noise from all NS-NS inspirals (before subtraction) is plotted in Fig. 2, alongside the noise level from the sought-for inflationary background and BBO's instrumental noise curve. Clearly, the NS-binary foreground has amplitude $\sim 10^{2}$ times higher than the (hypothetical) inflationary background's, in the BBO band, and so it must be possible to reduce (by subtraction) the foreground amplitude by more than $\sim 10^{2.5}$ to reveal an underlying primordial background.

Given our $r(z)$ and fiducial cosmological model, it is also straightforward to determine what fraction of $S_{\mathrm{h}}^{\mathrm{NSm}}(f)$ is due to sources farther out than some given redshift $z$. The result is plotted in Fig. 4. For example, 64\% of the foreground spectral density is due to sources at $z<1$, and $99 \%$ is due to sources merging at $z<3.6$. Thus, very roughly speaking, one must subtract out all NS-NS mergers up to $z \approx 3.6$ to reduce the foreground noise amplitude by one

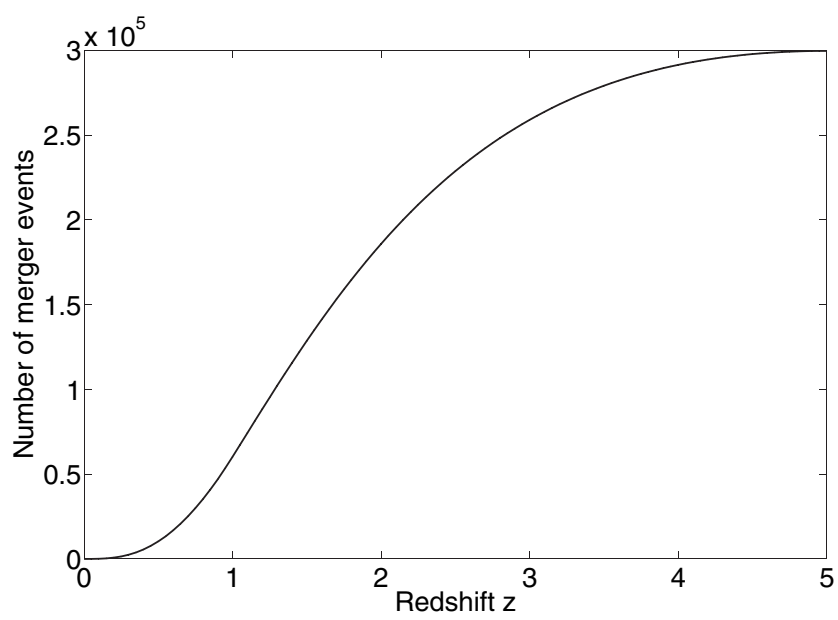

FIG. 3. The total number of NS-NS mergers closer than redshift $z$, The results here are normalized to a 3 yr observation period and $\dot{n}_{0}=10^{-7} \mathrm{Mpc}^{-3} \mathrm{yr}^{-1}$.

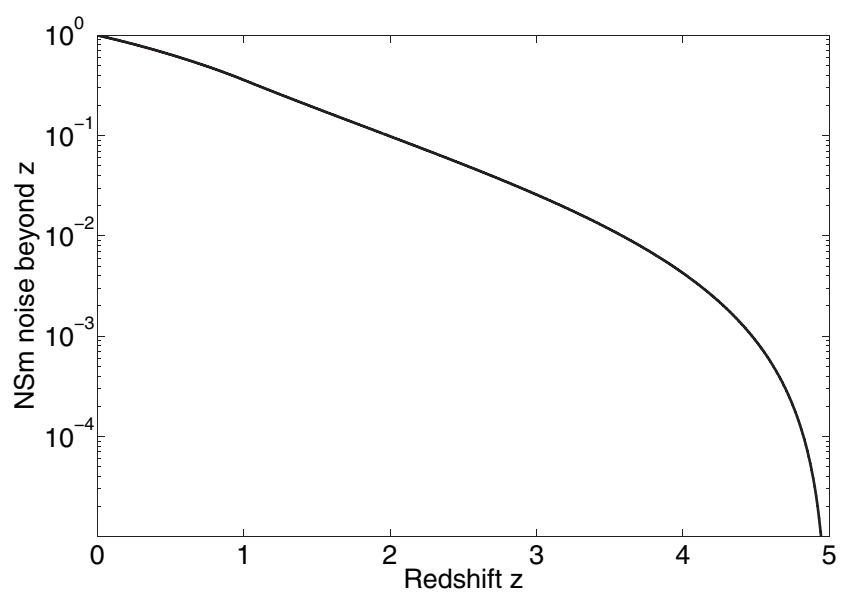

FIG. 4. Figure plots $S_{\mathrm{h}}^{\mathrm{NSm},>\mathrm{z}} / S_{\mathrm{h}}^{\mathrm{NSm}}$ vs $z$, i.e., it plots the fractional contribution of NS-NS binaries beyond redshift $z$ to the total NS-NS foreground noise.

order-of-magnitude. Of course, that conclusion is too simplistic, since the redshift out to which any particular NS binary can be observed depends on that binary's orientation as well as its redshift; see Sec. VI below for a proper accounting of this dependence.

\section{UNDERSTANDING CONFUSION NOISE: WHY NS-NS CHIRPS INTERFERE WITH EACH OTHER}

So far, we have computed a spectrum for the NS-NS inspiral foreground, but we have not yet explained in what sense this foreground represents a noise source for BBO. We do so in this section, showing how GW signals from different mergers "interfere with" and so obscure each other. In this paper we simply sketch the main results; full details will be provided elsewhere [19].

\section{A. Brief review of optimal matched filtering}

Typical NS-NS merger signals will have amplitudes roughly 2 orders of magnitude smaller than the amplitude of BBO's instrumental noise. In practice, therefore, (some version of) matched filtering will be required to dig these buried signals out of the noise. Hence we will begin by briefly reviewing optimal matched filtering, partly to fix notation. For a more complete discussion, see [20] or [21].

The output of $N$ detectors can be represented by the vector $s_{\mathrm{A}}(t), A=1,2, \ldots, N$. It is often convenient to work with the Fourier transform of the signal; the convention we use is

$$
\tilde{s}_{\mathrm{A}}(f) \equiv \int_{-\infty}^{\infty} e^{2 \pi i f t} s_{\mathrm{A}}(t) \mathrm{d} t
$$

The output $s_{\mathrm{A}}(t)$ is the sum of gravitational-wave signal $h_{\mathrm{A}}(t)$ plus instrumental noise $n_{\mathrm{A}}(t)$. In this section we will assume that the instrumental noise is both stationary and Gaussian. "Stationarity" essentially means that the different Fourier components $\tilde{n}_{\mathrm{A}}(f)$ of the noise are uncorre- 
lated; thus we have

$$
\overline{\tilde{n}_{\mathrm{A}}(f) \tilde{n}_{\mathrm{B}}\left(f^{\prime}\right)^{*}}=\frac{1}{2} \delta\left(f-f^{\prime}\right) S_{\mathrm{h}, \mathrm{AB}}(f),
$$

where an overline " - " denotes the "expectation value" and $S_{\mathrm{h}, A B}(f)$ is referred to as the spectral density of the noise. [When $N=1$ (i.e., when there is just a single detector), we will dispense with detector indices and just write $\tilde{s}(f)$ and $S_{\mathrm{h}}(f)$.] For our problem, we can restrict attention to the case where noises in different detectors are uncorrelated; then we have

$$
\overline{\tilde{n}_{\mathrm{A}}(f) \tilde{n}_{\mathrm{B}}\left(f^{\prime}\right)^{*}}=\frac{1}{2} \delta\left(f-f^{\prime}\right) S_{\mathrm{h}, \mathrm{A}}(f) \delta_{\mathrm{AB}}
$$

Given stationarity, "Gaussianity" implies that each Fourier component has Gaussian probability distribution. Under the assumptions of stationarity and Gaussianity, we obtain a natural inner product on the vector space of signals. Given two signals $g_{\mathrm{A}}(t)$ and $k_{\mathrm{A}}(t)$, we define $\langle\mathbf{g} \mid \mathbf{k}\rangle$ by

$$
\langle\mathbf{g} \mid \mathbf{k}\rangle=2 \sum_{\mathrm{A}} \int_{-\infty}^{\infty} \frac{\tilde{g}_{\mathrm{A}}^{*}(f) \tilde{k}_{\mathrm{A}}(f) \mathrm{d} f}{S_{\mathrm{h}, \mathrm{A}}(f)}
$$

It also follows from Eqs. (21) and (22) that for any functions $g_{\mathrm{A}}(t)$ and $k_{\mathrm{A}}(t)$, the expectation value of $(\mathbf{g} \mid \mathbf{n})(\mathbf{k} \mid \mathbf{n})$, for an ensemble of realizations of the detector noise $n_{\mathrm{A}}(t)$, is just $(\mathbf{g} \mid \mathbf{k})$.

In terms of this inner product, the probability for the noise to have some realization $\mathbf{n}_{0}$ is just

$$
p\left(\mathbf{n}=\mathbf{n}_{0}\right) \propto e^{-\left\langle\mathbf{n}_{0} \mid \mathbf{n}_{0}\right\rangle / 2} .
$$

Thus, if the actual incident waveform is $\mathbf{h}$, the probability of measuring a signal $\mathbf{s}$ in the detector output is proportional to $e^{-\langle\mathbf{s}-\mathbf{h} \mid \mathbf{s}-\mathbf{h}\rangle / 2}$. Correspondingly, given a measured signal $\mathbf{s}$, the gravitational waveform $\mathbf{h}$ that "best fits" the data is the one that minimizes the quantity $\langle\mathbf{s}-\mathbf{h} \mid \mathbf{s}-\mathbf{h}\rangle$.

For a given incident gravitational wave, different realizations of the noise will give rise to somewhat different best-fit parameters. However, for large SNR, the best-fit parameters will have a Gaussian distribution centered on the correct values. Specifically, let $\tilde{\lambda}^{\alpha}$ be the "true" values of the physical parameters, and let $\tilde{\lambda}^{\alpha}+\Delta \lambda^{\alpha}$ be the bestfit parameters in the presence of some realization of the noise. Then for large SNR, the parameter-estimation errors $\Delta \lambda^{\alpha}$ have the Gaussian probability distribution

$$
p\left(\Delta \lambda^{\alpha}\right)=\mathcal{N} \mathrm{e}^{-(1 / 2) \Gamma_{\alpha \beta} \Delta \lambda^{\alpha} \Delta \lambda^{\beta}}
$$

Here $\Gamma_{\alpha \beta}$ is the so-called Fisher information matrix defined by

$$
\Gamma_{\alpha \beta} \equiv\left\langle\frac{\partial \mathbf{h}}{\partial \lambda^{\alpha}} \mid \frac{\partial \mathbf{h}}{\partial \lambda^{\beta}}\right\rangle
$$

and $\mathcal{N}=\sqrt{\operatorname{det}(\Gamma / 2 \pi)}$ is the appropriate normalization factor. For large SNR, the variance-covariance matrix is given by

$$
\overline{\Delta \lambda^{\alpha} \Delta \lambda^{\beta}}=\left(\Gamma^{-1}\right)^{\alpha \beta}+\mathcal{O}(\mathrm{SNR})^{-1} .
$$

In the above notation, optimal filtering for some gravitational waveform $h(t)$ simply amounts to taking the inner product of $h(t)$ with the data stream $s(t)$. Assuming $\mathbf{s}=$ $\mathbf{n}+\mathbf{h}$, then

$$
\langle\mathbf{s} \mid \mathbf{h}\rangle=\langle\mathbf{n} \mid \mathbf{h}\rangle+\langle\mathbf{h} \mid \mathbf{h}\rangle .
$$

The first term on the right-hand side (rhs) of Eq. (25) has rms value $\langle\mathbf{h} \mid \mathbf{h}\rangle^{1 / 2}$, so the signal-to-noise of the detection will be approximately given by

$$
\operatorname{SNR}[\mathbf{h}]=\frac{\langle\mathbf{h} \mid \mathbf{h}\rangle}{\operatorname{rms}\langle\mathbf{h} \mid \mathbf{n}\rangle}=\langle\mathbf{h} \mid \mathbf{h}\rangle^{1 / 2} .
$$

\section{B. Overlapping NS-NS chirps as a source of self-confusion}

Now imagine that the detector output $s(t)$ consists of instrumental noise $n(t)$ plus the sum of some large number of merger signals ( labeled by " $i$ "):

$$
s(t)=n(t)+\sum_{i} h_{i}(t)
$$

(For simplicity, here we will consider the case of a single detector, and so eliminate the index $A$; the generalization to multiple detectors is trivial.)

As explained above, optimally filtering the data for any particular merger waveform $h_{j}(t)$ is equivalent to taking the inner product $\left\langle\mathbf{s} \mid \mathbf{h}_{j}\right\rangle$, which we can write as the sum of three pieces:

$$
\left\langle\mathbf{s} \mid \mathbf{h}_{j}\right\rangle=\left\langle\mathbf{n} \mid \mathbf{h}_{j}\right\rangle+\sum_{i \neq j}\left\langle\mathbf{h}_{i} \mid \mathbf{h}_{j}\right\rangle+\left\langle\mathbf{h}_{j} \mid \mathbf{h}_{j}\right\rangle .
$$

For the signal to be detectable, the third term should be significantly larger than the rms values of the first and second terms. We now explain why the second term can be sizeable; i.e., why different chirp signals can have substantial overlaps ${ }^{1}$. To simplify this discussion, let us use a slightly simpler version of the inner product; define

$$
(\mathbf{g} \mid \mathbf{k}) \equiv \int_{-\infty}^{\infty} \tilde{g}^{*}(f) \tilde{k}(f) \mathrm{d} f=\int_{-\infty}^{\infty} g(t) k(t) \mathrm{d} t,
$$

where the second equality in Eq. (29) is just Parseval's theorem. (Clearly, this is just our usual inner product, but without the "reweighting by $1 / S_{\mathrm{h}}$ " in the frequency domain. For white noise, where $S_{\mathrm{h}}(f)=$ const., $(\mid)$ and $\langle\mid\rangle$ are equivalent, except for an overall constant.)

We now want to estimate the values of $\left(\mathbf{n} \mid \mathbf{h}_{i}\right)$ and $\left(\mathbf{h}_{i} \mid \mathbf{h}_{j}\right)$ for any two binary inspiral waveforms $h_{i}(t)$ and $h_{j}(t)$. In

\footnotetext{
${ }^{1}$ One of the authors, C.C., presented this analysis in Dec., 2001 at GWDAW2001 in Trento, Italy, but heretofore had not published it.
} 
the nearly-Newtonian regime of interest to $\mathrm{BBO}$, these are simple chirp waveforms:

$$
\begin{aligned}
& h_{i}(t)=A_{i}(t) \cos \Phi_{i}(t), \\
& h_{j}(t)=A_{j}(t) \cos \Phi_{j}(t),
\end{aligned}
$$

where

$$
\begin{aligned}
& \Phi_{i}(t)=\cos \int^{t} 2 \pi f_{i}\left(t^{\prime}\right) \mathrm{d} t^{\prime}, \\
& \Phi_{j}(t)=\cos \int^{t} 2 \pi f_{j}\left(t^{\prime}\right) \mathrm{d} t^{\prime},
\end{aligned}
$$

and where $A_{i}(t), A_{j}(t), f_{i}(t)$, and $f_{j}(t)$ are all slowly varying (meaning their fractional change during one cycle is $\ll$ $1)$, and $f_{i}(t)$ and $f_{j}(t)$ are monotonically increasing. Then, since the integrand is highly oscillatory, it is clear that the integral $\int h_{i}(t) h_{j}(t) \mathrm{d} t$ will show substantial waveform overlap only if there is some instant $t_{0}$ when the two signals have the same frequency:

$$
f_{i}\left(t_{0}\right)=f_{j}\left(t_{0}\right),
$$

i.e., if one considers the "track" of each signal in the $f-t$ plane, then $t_{0}$ is the instant of time when the two tracks cross. Using the stationary-phase approximation, it is straightforward to show that [19]:

$$
\left(\mathbf{h}_{i} \mid \mathbf{h}_{j}\right) \approx \frac{1}{2} A_{i}\left(t_{0}\right) A_{j}\left(t_{0}\right)|\delta \dot{f}|^{-1 / 2} \cos \left[\Delta \Phi_{0} \pm \pi / 4\right],
$$

where $\Delta \Phi_{0} \equiv\left[\Phi_{i}\left(t_{0}\right)-\Phi_{j}\left(t_{0}\right)\right], \quad \delta \dot{f} \equiv\left[\dot{f}_{i}\left(t_{0}\right)-\dot{f}_{j}\left(t_{0}\right)\right]$, and where the sign in front of the $\pi / 4$ in Eq. (35) is positive when $\delta \dot{f}>0$ and negative when $\delta \dot{f}<0$.

We want to use this result to estimate

$$
\left(\mathbf{h}_{j} \mid \sum_{i \neq j} \mathbf{h}_{i}\right)
$$

i.e., to sum the contributions from all binaries whose $f-t$ tracks overlap the $j^{\text {th }}$ track. Since the phase differences $\Delta \Phi_{0}$ at different intersections will clearly be uncorrelated, the contributions accumulate in a random-walk fashion; i.e., the square of the sum is approximately the sum of the squares of the individual terms. Also, as we show in the next subsection, a typical NS-NS track will intersect a very large number of tracks from other merging binaries, so we are in the realm of large-number statistics. Finally, while the magnitude of each squared-contribution scales like $|\delta \dot{f}|^{-1}$, the number of terms in the sum scales like the average value of $|\delta \dot{f}|$, since the larger the "relative velocities" of the tracks, the more crossings. The dependence of the sum on the typical size of $|\delta \dot{f}|$ therefore ends up cancelling out, and one can show the following [19]. Let $H(t)=\sum_{i} h_{i}(t)$ be the entire foreground generated by NSNS chirps, and let $H$ 's spectral density be $S_{\mathrm{H}}(f)$, normalized so that

$$
\overline{H^{2}(t)}=\int_{0}^{\infty} S_{\mathrm{H}}(f) \mathrm{d} f .
$$

Then the expectation value of $\left(\mathbf{h}_{j} \mid \sum_{i \neq j} \mathbf{h}_{i}\right)^{2}$ is given by

$$
\overline{\left(\mathbf{h}_{j} \mid \sum_{i \neq j} \mathbf{h}_{i}\right)^{2}}=\frac{1}{2} \int h_{j}^{2}(t) S_{\mathrm{H}}\left(f_{j}(t)\right) \mathrm{d} t .
$$

But the same result holds for the mean-square overlap of $h_{j}(t)$ with stationary, Gaussian noise $n(t)$ :

$$
\overline{\left(\mathbf{h}_{j} \mid \mathbf{n}\right)^{2}}=\frac{1}{2} \int h_{j}^{2}(t) S_{\mathrm{h}}\left(f_{j}(t)\right) \mathrm{d} t
$$

with $\overline{n^{2}(t)}=\int_{0}^{\infty} S_{\mathrm{h}}(f) \mathrm{d} f$; i.e., the mean-square overlap of a single chirp $h_{j}(t)$ with the chirp foreground $H(t)$ (excluding $h_{j}$ itself) is the same as the mean-square overlap of $h_{j}(t)$ with stationary, Gaussian noise having the same spectral density as $H$. (It is straightforward to generalize this result to inner products with nontrivial frequencyweighting [19].) It is for this reason that in Eq. (1) we simply add together the spectral densities of the instrumental noise and the "confusion noise" from unresolved chirps.

\section{The number of overlapping inspiral tracks in the $f-t$ plane}

We saw in the previous subsection that two chirp signals have substantial overlap only if their tracks in the $f-t$ plane intersect. Here we consider the track from a typical NS-NS inspiral and estimate how many other inspiral tracks it crosses.

Let $\rho(f)$ be the probability density of merger signals in frequency space; i.e., at any instant, $\rho(f) \Delta f$ is the average number of NS-NS GW signals received near the Earth that are in the frequency range $[f-\Delta f / 2, f+\Delta f / 2]$. Since the $\mathrm{BBO}$ mission lifetime is vastly shorter than the age of the universe, we can assume $\rho(f)$ is time-independent, implying

$$
\rho(f) \frac{\mathrm{d} f}{\mathrm{~d} t}=\text { const. }=\dot{N},
$$

where, again, $\dot{N} \equiv \Delta N_{\mathrm{m}} / \Delta \tau_{0}$ is the total rate of mergers in the observable universe (from all $z$ ). The GW frequency derivative $\dot{f}$ is given by

$$
\mathrm{d} f / \mathrm{d} t=\frac{96}{5} \pi^{8 / 3}[\mathcal{M}(1+z)]^{5 / 3} f^{11 / 3} .
$$

so clearly $\rho(f) \propto f^{-11 / 3}$.

Now consider any one track in the $f-t$ plane, and examine it in the neighborhood of some frequency $f$. It is easy to see that the rms rate $r_{\mathrm{c}}$ at which it intersects neighboring tracks is

$$
r_{\mathrm{c}}=0.5 \rho(f) \Delta \dot{f},
$$

where $\Delta \dot{f}$ is the rms variation in frequency derivatives for 
sources with GW frequency $f$. The 0.5 factor in Eq. (42) arises because, for any two neighboring tracks at any instant, there is a 50\% chance that they are approaching each other and a 50\% chance that they are separating.

Using Eq. (41), we see that the rms relative "velocity" of nearby tracks is

$$
\frac{\Delta \dot{f}}{\dot{f}}=5 / 3 \frac{\Delta \mathcal{M}_{\mathrm{eff}}}{\mathcal{M}_{\mathrm{eff}}}
$$

where we define $\mathcal{M}_{\text {eff }} \equiv \mathcal{M}(1+z)$, and where $\Delta \mathcal{M}_{\text {eff }}$ is the rms variation in this quantity. Now the fractional variation in $\mathcal{M}$ itself, $\Delta \mathcal{M} / \mathcal{M}$, is probably at least of order 0.1 . However, from Fig. 3 we see that this is small compared to the variation $\Delta(1+z) /(1+z)$, which is $\sim 0.4$.

Thus $r_{\mathrm{c}}$ is roughly given by

$$
r_{\mathrm{c}}=0.5(5 / 3) \rho \dot{f} \frac{\Delta \mathcal{M}_{\mathrm{eff}}}{\mathcal{M}_{\mathrm{eff}}} \sim \frac{1}{3} \dot{N}
$$

independent of the particular frequency $f$. That is, the rate at which any particular track crosses all other tracks is about one-third the total merger rate from all observable sources, independent of where one is on the track. Thus, for any one track over the last 3 years of inspiral, one expects of order $10^{5}$ crossings. This amply justifies our use of large-number statistics in the previous subsection.

\section{CONFUSION NOISE FROM IMPERFECTLY SUBTRACTED WAVEFORMS}

NS binaries limit BBO's sensitivity to a primordial background in two ways. First, there will be some binaries that are too weak (because of their distance and/or orientation) to be individually identified and subtracted, and these "unidentified binaries" clearly represent a source of "confusion noise." Second, even identified NS binaries will not be removed perfectly from the data stream; inevitably (due to the finite signal-to-noise of the observations) there are subtraction errors, which represent a second source of confusion noise. This section addresses the confusion noise that results from subtraction errors. First we will prove a simple theorem regarding the magnitude of subtraction errors. Then we will sketch a simple strategy for largely eliminating their impact on other analyses by projecting them out, at the cost of some bandwidth. We estimate that lost bandwidth for BBO, and conclude that the loss is small enough that in the rest of this paper we can safely neglect it.

We believe the analysis and strategy we outline here will also be useful in similar contexts, especially in dealing with problems of confusion noise in LISA data. Here we provide only a sketch of the main ideas; more details will be provided in a forthcoming publication [19].

\section{A. Subtraction errors due to noise}

We have argued that, before searching for a primordial GW background, one will want to first subtract from the data the best fit to each identified inspiralling compact binary. However, because of detector noise, the best-fit values of the binary parameters will differ from their true values, and so the best-fit waveforms will be somewhat in error. What is the typical size of the error? That is easy to calculate: Let $h(t)$ be some gravitational waveform immersed in noisy data, and assume the waveform depends on $N_{\mathrm{p}}$ physical parameters $\lambda^{\alpha}\left(\alpha=1, \cdots, N_{\mathrm{p}}\right)$. Because of the noise, the best-fit parameter values $\hat{\lambda}^{\alpha}$ will differ from the true parameter values by [20]

$$
\delta \lambda^{\alpha} \equiv \hat{\lambda}^{\alpha}-\lambda^{\alpha} \approx\left(\Gamma^{-1}\right)^{\alpha \beta}\left\langle\mathbf{n} \mid \partial_{\beta} \mathbf{h}\right\rangle,
$$

and, correspondingly, the best-fit waveform $\hat{h}(t)$ will differ from the true one by

$$
\delta \mathbf{h} \equiv \hat{\mathbf{h}}-\mathbf{h}=\partial_{\alpha} \mathbf{h} \delta \lambda^{\alpha}+\mathcal{O}(\delta \lambda)^{\mathbf{2}} .
$$

Using Eqs. (23), (24), and (46), we can immediately estimate the norm-squared of this residual error. To lowest order in $\delta \lambda^{\alpha}$, we have

$$
\overline{\langle\delta \mathbf{h} \mid \delta \mathbf{h}\rangle}=\left\langle\partial_{\alpha} \mathbf{h} \mid \partial_{\beta} \mathbf{h}\right\rangle \overline{\delta \lambda^{\alpha} \delta \lambda^{\beta}}=\Gamma_{\alpha \beta}\left(\Gamma^{-1}\right)^{\alpha \beta}=N_{\mathrm{p}} .
$$

Thus the size of $\langle\delta \mathbf{h} \mid \delta \mathbf{h}\rangle$ is independent of the signal strength, but increases linearly with the number of parameters that need to be fit for.

Equation (47) estimates the weighted integral of $|\delta \tilde{h}(f)|^{2}$; it says nothing about rms size of $|\delta \tilde{h}(f)|^{2}$ at any particular frequency $f$. Now, one can always calculate $|\delta \tilde{h}(f)|^{2}$ using (to lowest order)

$$
|\delta \tilde{h}(f)|^{2}=\partial_{\alpha} \tilde{h}(f) \partial_{\beta} \tilde{h}^{*}(f)\left(\Gamma^{-1}\right)^{\alpha \beta},
$$

but for back-of-the-envelope calculations, it is reasonable to simply turn Eq. (47) into a point estimate for the relative error:

$$
\frac{|\delta \tilde{h}(f)|}{|\tilde{h}(f)|} \sim\left[\frac{\langle\delta \mathbf{h} \mid \delta \mathbf{h}\rangle}{\langle\mathbf{h} \mid \mathbf{h}\rangle}\right]^{1 / 2} \sim \frac{N_{\mathrm{p}}^{1 / 2}}{\mathrm{SNR}} .
$$

For BBO measurements of NS-NS binaries, $N_{\mathrm{p}} \approx 11$ (cf. Sec. VI), and for a typical source (i.e, for a source at $z \approx$ 1.5 , with $\mu=0.5$, where $\mu$ is the cosine of the angle between the line-of-sight and the normal to the binary's orbital plane), $\mathrm{SNR} \approx 140$, so $\delta h / h \sim 2.4 \times 10^{-2}$.

Given the extreme accuracy with which foreground sources must be subtracted, at first glance this level of error seems unacceptable. However it would be a mistake to regard $\delta h$ as a completely random, additive noise source in the data. For one thing, after the best-fit signal $\hat{h}(t)$ has been removed from the data stream, the amplitude of noise plus residual is smaller (on average) than that of the noise alone. To see this, consider again the case of data $s(t) \equiv$ $n(t)+h(t)$, and assume that the observation time is $T$, and 
that the data has been band-limited to $\left[-f_{\max }, f_{\max }\right]$. Then it is easy to show that the noise alone has squaredmagnitude:

$$
\overline{\langle\mathbf{n} \mid \mathbf{n}\rangle}=2 f_{\max } T,
$$

which is just the number of data points, for data sampled at the Nyquist rate $2 f_{\max }$. Next consider the magnitudesquared of the post-subtraction data set, $\mathbf{s}-\hat{\mathbf{h}}$, where again $\hat{\mathbf{h}}$ is the waveform that best fits the data. A straightforward calculation shows that [19]

$$
\begin{gathered}
\overline{\langle\mathbf{s}-\hat{\mathbf{h}} \mid \mathbf{s}-\hat{\mathbf{h}}\rangle}=\overline{\langle\mathbf{n}-\delta \mathbf{h} \mid \mathbf{n}-\delta \mathbf{h}\rangle} \\
=2 f_{\max } T-N_{\mathrm{p}},
\end{gathered}
$$

i.e., fitting out waveform $\hat{h}$ causes the norm-squared of the data to decrease below what is expected from noise alone. This is easy to understand: the fitting procedure takes out not only the signal $\mathbf{h}$, but also that part of the noise that "looks like" the difference between $\mathbf{h}$ and some other waveform, $\hat{\mathbf{h}}$, having slightly different physical parameters. Stated geometrically, if one considers the manifold of physical gravitational waveforms, embedded in the vector space of possible measured signals, one sees that any piece of the noise that is tangent to the waveform manifold (at the location of the true signal) gets fitted out. Indeed, one sees from Eq. (45) that it is just this piece of the noise, lying in the tangent space to the waveform manifold, that is "responsible" for the parameter-estimation errors $\delta \lambda^{\alpha}$ in the first place. In the next subsection we outline a strategy projecting out this error before one searches for an inflation-generated background.

Note that nothing in the above arguments required the signal to emanate from a single physical source; e.g., if $H(t)$ is the entire foreground signal coming from $N_{\mathrm{s}}$ sources,

$$
H(t)=\sum_{i=1}^{N_{\mathrm{s}}} h_{i}(t)
$$

and if each $h_{i}(t)$ is described by $p$ parameters, then the full parameter space is described by $N_{\mathrm{p}}=p \times N_{\mathrm{s}}$ parameters, and

$$
\overline{\langle\delta \mathbf{H} \mid \delta \mathbf{H}\rangle}=p \times N_{\mathrm{s}}
$$

to lowest order in $1 / \mathrm{SNR}$. The total $\mathrm{SNR}^{2}$ of the foreground $H$ is just $N_{\mathrm{s}}$ times the average $\mathrm{SNR}^{2}$ of the individual sources, and $N_{\mathrm{p}}$ is of course directly proportional to $N_{\mathrm{s}}$, so the fractional error in subtracting the whole foreground is just the fractional error in subtracting a typical source:

$$
\delta H / H \sim \delta h / h .
$$

For BBO measurements of the NS-NS foreground, we thus estimate $\delta H / H \sim 2.4 \times 10^{-2}$.
As a digression, we remark that because our foreground consists of a large number of overlapping sources, it should not be surprising if there are some near degeneracies that make it practically impossible to determine some of the physical parameters of some of the sources. (These are cases where the affect on $H(t)$ of adjusting the parameters of one source can be almost perfectly cancelled by adjusting the parameters of another source.) We bring this up to make the point that such near degeneracies do not necessarily imply any degradation in one's ability to subtract out the foreground. Indeed, in the case of very high SNR (per source), it implies the opposite: near degeneracies would imply that the residual $\delta \mathbf{H}$ is somewhat smaller than estimated above. The reason is simple: a near degeneracy means that the effective dimensionality of the signal space (near the actual signal) is smaller than the number of parameters being used to describe it; i.e., one could find a new parametrization using a fewer number of variables, $N_{\mathrm{p}}^{\prime}$. Then a repetition of the above arguments would yield $\overline{\langle\delta \mathbf{H} \mid \delta \mathbf{H}\rangle}=N_{\mathrm{p}}^{\prime}<N_{\mathrm{p}}$. For the BBO case, where SNR per source is $\approx 140$, it probably will require detailed simulations to determine whether subtraction errors are larger or smaller than indicated by the high-SNR result, Eq. (54). We leave this question to future work.

\section{B. Projecting out residual subtraction errors}

In this subsection we propose one strategy for effectively cleaning the BBO data of subtraction errors, after the NSNS binaries have been subtracted out. Using this strategy, we argue that the impact of subtraction residuals (arising from instrumental noise) becomes sufficiently small that they can be ignored in the rest of this paper. We do not argue that our strategy is the best one possible, but rather offer it as an "existence argument" that some such strategy is possible. The use of any alternative strategy that leads to the same conclusion would not affect the main results of this paper.

The basic observation behind our strategy is that the residual $\delta H(t)$ is mostly confined to a surface within the vector space of all signals: the tangent space to the waveform manifold at the best-fit point. The corresponding errors in the subtracted waveform can be expanded in a Taylor series:

$$
\delta H(t)=\partial_{\alpha} H(t) \delta \lambda^{\alpha}+\frac{1}{2} \partial_{\alpha} \partial_{\beta} H(t) \delta \lambda^{\alpha} \delta \lambda^{\beta}+\cdots,
$$

where $\alpha, \beta=1, \ldots, p \times N_{\mathrm{s}}$. The first-order piece on the rhs is the linear combination of $N_{\mathrm{p}}=p \times N_{\mathrm{s}}$ wavefunctions (the $\partial_{\alpha} H(t)$ ), with unknown coefficients (determined by the noise). We propose projecting these directions out of the data stream. This is simple in principle. Consider the operator

$$
P \equiv I-\left(\Gamma^{-1}\right)^{\alpha \beta}\left|\partial_{\alpha} \mathbf{H}\right\rangle\left\langle\partial_{\beta} \mathbf{H}\right|,
$$

where for simplicity we use here standard bra-ket notation 
of quantum mechanics. It is trivial to verify that $P^{2}=P$ and that $P$ destroys any wavefunction of the form $\partial_{\alpha} H(t) \delta \lambda^{\alpha}$. We propose acting on the data streams with $P$ before searching them for an inflation-generated background.

What fraction of the data have we thrown away, by using $P$ ? For a fiducial $3 \mathrm{yr}$ BBO lifetime, with, say, $\sim 3 \times 10^{5}$ subtracted sources, each determined by $\sim 11$ parameters, $N_{\mathrm{p}} \sim 3 \times 10^{6}$. Assuming a $2 \mathrm{~Hz}$ sampling rate (sufficient for capturing most of the signal), with $\sim 10^{8} \mathrm{~s}$ of data and 8 independent channels, the dimension $S$ of the full data space is $S \sim 1.5 \times 10^{9}$. Thus the fraction of the data that is discarded is only $N_{\mathrm{p}} / S \sim 2 \times 10^{-3}$, which is a negligible loss.

So far we have discussed projecting out the first-order piece of the subtraction error; i.e., the piece linear in the parameter-estimation errors $\delta \lambda^{\alpha}$. What is the magnitude of the second-order subtraction errors (i.e., the ones quadratic in $\left.\delta \lambda^{\alpha}\right)$ ? This is clearly given by

$$
\overline{\left\langle\delta^{2} \mathbf{H} \mid \delta^{2} \mathbf{H}\right\rangle}=\frac{1}{4}\left\langle\partial_{\alpha} \partial_{\beta} \mathbf{H} \mid \partial_{\gamma} \partial_{\epsilon} \mathbf{H}\right\rangle \overline{\delta \lambda^{\alpha} \delta \lambda^{\beta} \lambda^{\gamma} \delta \lambda^{\epsilon}},
$$

but evaluating the rhs of Eq. (58) is beyond the scope of this paper, and so we content ourselves with a cruder estimate. The second-order errors clearly scale like the square of the first-order errors, so a very crude estimate is $\delta^{2} H / H \sim(\delta H / H)^{2} \sim 6 \times 10^{-4}$. Of course, this estimate is properly multiplied by some prefactor (which can only be obtained by calculating of the rhs of Eq. (58)). Depending on this prefactor and the actual level of the NSNS foreground, these second-order subtraction errors could be comparable in size to the sought-for inflationary background. If this is the case, we would advocate projecting out the second-order errors as well. The second-order errors are linear combinations of second derivatives $\partial_{\alpha} \partial_{\beta} H(t)$. It is important to notice that such second derivatives vanish identically unless $\alpha$ and $\beta$ are parameters describing the same binary. Thus the vast majority of such second derivatives vanish. For each binary, there are $(11 \times$ 12) $/ 2=66$ nonvanishing second derivatives, so projecting out the second-order piece of the subtraction errors would cost only $\sim 1 \%$ of BBO's bandwidth. A crude estimate of the size of third-order subtraction errors is $\delta^{3} H / H \sim$ $(\delta H / H)^{3} \sim 10^{-5}$. Clearly, unless the missing prefactor here is quite large (of order 100 or more), it should not be necessary to project these third-order errors out of the data.

\section{CATALOG OF RELEVANT PHYSICAL PARAMETERS AND RELEVANT EFFECTS}

\section{A. Subtraction errors due to inaccurate waveform templates}

In the previous section, we outlined a method for handling subtraction errors arising from instrumental noise. Another potential source of subtraction error is inaccurate theoretical template waveforms. Provisionally, we will regard a physical parameter, effect, or post-Newtonian term as "relevant for BBO" if neglecting it would lead to relative errors in our theoretical inspiral waveforms of size $\delta h / h \gtrsim 10^{-3}$ (since errors of that magnitude could dominate over the inflationary background). Since each inspiral waveform contains $\sim 10^{7}$ cycles, knowing the waveforms to $\delta h / h \geqslant 10^{-3}$ requires calculating the waveform phase to roughly one part in $10^{11}$.

The post-Newtonian (PN) expansion is clearly the right tool for constructing the waveforms, since the PN expansion parameter $M / r$ is small in the $\mathrm{BBO}$ band:

$$
\frac{M}{r} \approx 5.5 \times 10^{-4}\left(\frac{M[1+z]}{2.8 M_{\odot}}\right)^{2 / 3}\left(\frac{f}{0.3 \mathrm{~Hz}}\right)^{2 / 3},
$$

where $f$ is the GW frequency. If one uses PN waveforms, the only reasons for theoretical error would be (1) failure to calculate post-Newtonian corrections to sufficiently high order in the PN expansion, or (2) failure to account for all relevant physical parameters (e.g., the spins of the NSs).

This section provides an initial "scoping out" of the questions of which physical parameters are relevant, and which post-Newtonian order is sufficient.

\section{B. Orbital eccentricity}

\section{Typical eccentricities of binaries in the $\mathrm{BBO}$ band}

Here we consider the implications of small (but nonzero) eccentricity for the subtraction problem. We begin by estimating typical eccentricities of NS binaries when they are emitting GWs in the $\mathrm{BBO}$ band.

It is well known that radiation reaction tends to circularize the orbits of nearly-Newtonian binaries. For small eccentricity $e, e^{2}$ decreases with the orbital period $P$ according to $e^{2} \propto P^{19 / 9}$ [22]. For arbitrary $e$, the mutual scaling is given by [22]

$$
P^{2 / 3} \propto \frac{e^{12 / 19}}{\left(1-e^{2}\right)}\left[1+\frac{121}{304} e^{2}\right] .
$$

The two known NS-NS binaries that dominate current merger rate estimates are PSR $1913+16$ and PSR J07373039. Extrapolating from today's values of $e$ and $P$ for these two binaries, using Eq. (63), we estimate that their eccentricities when they pass through the $\mathrm{BBO}$ band will be $e_{1913}^{2} \approx 4.6 \times 10^{-8}\left(\frac{f}{0.3 \mathrm{~Hz}}\right)^{-19 / 9}$ and $e_{0737}^{2} \approx 2.0 \times$ $10^{-9}\left(\frac{f}{0.3 \mathrm{~Hz}}\right)^{-19 / 9}$. Based on these two examples, we will provisionally assume that typical eccentricities are $e^{2} \sim$ $\left[10^{-9}-10^{-7}\right]\left(\frac{f}{0.3 \mathrm{~Hz}}\right)^{-19 / 9}$. However we will also consider the implications of a subpopulation of NS binaries with considerably larger eccentricity.

\section{Effect of nonzero eccentricity on waveform phase}

The effect of small, nonzero eccentricity is to slightly increase the inspiral rate; to lowest nontrivial PN order and 
to first order in $e^{2}$, the increase (derivable from [22]) is given by

$$
\mathrm{d} f / \mathrm{d} t=\mathrm{d} f /\left.\mathrm{d} t\right|_{e=0}\left[1+\frac{157}{24} e^{2}\right] .
$$

In the stationary-phase approximation, we can write the Fourier transform of the emitted waveform (omitting tensor indices) as [20]

$$
\tilde{h}(f) \propto(\mathcal{M}(1+z))^{5 / 6} f^{-7 / 6}[1+\ldots] \mathrm{e}^{\mathrm{i} \Psi(f)},
$$

where “..." stands for higher-order PN corrections, and where the phase $\Psi(f)$ can be written as

$$
\Psi(f)=\Psi_{0}(f)+\Psi_{\mathrm{e}}(f) .
$$

Here $\Psi_{0}(f)$ represents the zero-eccentricity phase evolution and has the following PN expansion:

$$
\begin{aligned}
\Psi_{0}(f)= & \text { const. }+2 \pi f t_{\mathrm{c}}+\frac{3}{4}(8 \pi \mathcal{M}(1+z) f)^{-5 / 3} \\
& \times\left[1+\frac{20}{9}\left(\frac{743}{336}+\frac{11 \mu}{4 M}\right) y-16 \pi y^{3 / 2}+\ldots\right] .
\end{aligned}
$$

with $y \equiv(\pi M(1+z) f)^{2 / 3}$, while $\Psi_{\mathrm{e}}(f)$ represents the phase correction due to nonzero $e^{2}$, and is given (again, to lowest nontrivial PN order and to first order in $e^{2}$ ) by [23]

$$
\Psi_{e}(f)=-\frac{7065}{187136}[\pi \mathcal{M}(1+z)]^{-5 / 3} e_{0}^{2} f_{0}^{19 / 9} f^{-34 / 9} .
$$

Here $e_{0}$ is the binary's eccentricity at the moment that the GW frequency (more specifically, the frequency of the dominant, $n=2$ harmonic) sweeps through some fiducial frequency $f_{0}$. (Note that, by Eq. (60), the combination $e_{0}^{2} f_{0}^{19 / 9}$ is a constant, to lowest nontrivial order.)

Plugging in fiducial values, we can reexpress Eq. (65) as

$$
\Psi_{\mathrm{e}}(f)=-0.21\left[\frac{e_{0.3 \mathrm{~Hz}}^{2}}{10^{-8}}\right]\left[\frac{(1+z) \mathcal{M}}{1.22 M_{\odot}}\right]^{-5 / 3}\left[\frac{f}{0.3 \mathrm{~Hz}}\right]^{-34 / 9} .
$$

Note the very steep falloff of $\Psi_{\mathrm{e}}(f)$ with increasing $f$. This $f^{-34 / 9}$ falloff is much steeper than for the other PN correction terms in Eq. (67), so it seems quite unlikely that errors in fitting for $e_{0}$ could be "absorbed" into compensating errors in the other parameters. While $\Psi_{\mathrm{e}}(f)$ is negligible for frequencies above a few $\mathrm{Hz}$, it is typically of size $\sim 2 \pi$ at $f=0.1 \mathrm{~Hz}$. Clearly, then, orbital eccentricity is a relevant parameter that must be accounted for, both in subtracting out individual sources and in projecting out residual errors. From Eq. (71), we can also estimate roughly how accurately BBO can measure the eccentricity of each binary; it should be possible to determine $e_{0.3 \mathrm{~Hz}}^{2}$ to within $\Delta\left(e_{0.3 \mathrm{~Hz}}^{2}\right) \sim\left[10^{-8} / \mathrm{SNR}\right] \sim 10^{-10}$.

\section{Contribution of $n=3$ radiation to $\Omega_{\mathrm{GW}}^{\mathrm{NSm}}$}

Nonzero orbital eccentricity implies that even the quadrupole piece of the gravitational radiation is no longer purely sinusoidal, but exhibits harmonics at all multiples $n \nu$ of the orbital frequency $\nu$ (for integers $n \geq 1$ ). Let $\dot{E}_{\mathrm{n}}$ be the gravitational luminosity due to the $n^{\text {th }}$ harmonic. For small $e, \dot{E}_{\mathrm{n}} \propto e^{|2 n-4|}$, so in the range of interest for $e$, only $\dot{E}_{3}$ and $\dot{E}_{1}$ could potentially be significant. While both $\dot{E}_{3}$ and $\dot{E}_{1}$ are $\propto e^{2}$, it is easy to show that the $n=3$ contribution to $\Omega_{\mathrm{GW}}^{\mathrm{NSm}}$ dominates over the $n=1$ contribution. Therefore we concentrate here on the $n=3$ harmonic.

The ratio $\dot{E}_{3} / \dot{E}_{2}$ is [24]

$$
\dot{E}_{3} / \dot{E}_{2} \approx(3 / 2)^{6} e^{2},
$$

from which one easily derives

$$
\begin{aligned}
\Omega_{\mathrm{GW}}^{n \geq 3}(f) & =\left(\frac{3}{2}\right)^{6}\left\langle e_{2 f / 3}^{2}\right\rangle \Omega_{\mathrm{GW}}^{n=2}(2 f / 3) \\
& =\left(\frac{3}{2}\right)^{6}\left(\frac{3}{2}\right)^{13 / 9}\left\langle e_{f}^{2}\right\rangle \Omega_{\mathrm{GW}}^{n=2}(f) \\
& \approx 1.6 \cdot 10^{-19}\left(\frac{n_{0}}{10^{3} \mathrm{Mpc}^{3}}\right)\left(\frac{\left\langle e_{0.3 \mathrm{~Hz}}^{2}\right\rangle}{10^{-8}}\right)\left(\frac{f}{0.3 \mathrm{~Hz}}\right)^{-13 / 9},
\end{aligned}
$$

where $\left\langle e_{0.3 \mathrm{~Hz}}^{2}\right\rangle$ is the average value (for all NS-NS mergers) of $e^{2}$ at $f=0.3 \mathrm{~Hz}$. For our fiducial estimate of $\left\langle e_{0.3 \mathrm{~Hz}}^{2}\right\rangle$, this is significantly below the sought-for level of inflationgenerated GWs, and so the extra harmonics generated by nonzero $e$ can be neglected.

However, our estimate that $\left\langle e_{0.3 \mathrm{~Hz}}^{2}\right\rangle \sim 10^{-8}$ was based on the few known examples of close NS-NS binaries; what if there is a subpopulation of NS-NS binaries that merge with substantially larger eccentricity (e.g., due to the Kozai mechanism [25])? The ratio of the $n=3$ to the $n=2$ piece of the waveform, $h^{n=3} / h^{n=2}$, is clearly of order $e$. Thus the $n=3$ piece must be subtracted (or projected out) if $e \geq 10^{-3}$. Fortunately, as the previous subsection makes clear, if $e_{0.3 \mathrm{~Hz}} \geqslant 10^{-5}$, then the waveform itself will inform us of this fact, via the phase evolution of the $n=2$ piece.

Unfortunately, to subtract $h^{n=3}$, one needs to know both $e$ and the perihelion angle $\omega$ (at some fiducial instant or frequency), since the latter clearly determines the relative phase of the $n=3$ and $n=2$ pieces. How accurately can $\omega_{0.3 \mathrm{~Hz}}$ be extracted from the data? Since $\omega$ is encoded only in the $n \neq 2$ harmonics, we estimate that $\Delta \omega_{03 \mathrm{~Hz}} \sim$ $\min \left\{\pi,\left(e_{0.3 \mathrm{~Hz}} \times \mathrm{SNR}\right)^{-1}\right\}$. Hence, while the $h^{n=3}$ piece is relevant for $e_{0.3 \mathrm{~Hz}} \gtrsim 10^{-3}$, it will be impossible to subtract it when $e_{0.3 \mathrm{~Hz}} \lesssim 10^{-2}$ (since $\omega_{0.3 \mathrm{~Hz}}$ will be undetermined). Fortunately, even in this case, $h^{n=3}$ can simply be projected out of the data (in the manner described in Sec. IV B) since all possible realizations of $h^{n=3}(t)$ lie in a two-dimensional vector space. To see this, note that if all parameters except $\omega_{0.3 \mathrm{~Hz}}$ were known, then one could 
express $h^{n=3}(t)$ in the form $A_{3}(t) \cos \left[3\left(\Phi_{3}(t)+\omega_{0.3 \mathrm{~Hz}}\right)\right]$, where $A_{3}(t)$ and $\Phi_{3}(t)$ are both known functions, and this can be expanded as $\cos \left[3 \omega_{0.3 \mathrm{~Hz}}\right] \times A_{3}(t) \cos \left[3 \Phi_{3}(t)\right]-$ $\sin \left[3 \omega_{0.3 \mathrm{~Hz}}\right] \times A_{3}(t) \sin \left[3 \Phi_{3}(t)\right]$; i.e., $h^{n=3}(t)$ is just some linear combination of two known waveforms, with (unknown) coefficients $\cos \left[3 \omega_{0.3 \mathrm{~Hz}}\right]$ and $\sin \left[3 \omega_{0.3 \mathrm{~Hz}}\right]$.

\section{Summary of effects of orbital eccentricity}

Extrapolating from the known NS-NS binaries, we have estimated that typical eccentricities for NS-NS binaries radiating in the $\mathrm{BBO}$ band will be $e \lesssim 10^{-4}$. At this level, they would have a significant impact on the phase evolution of the $n=2$ harmonic, but the $n=3$ and $n=1$ pieces of the waveform would be negligibly small. In this case, when projecting out residual errors, one need not worry about the perihelion angle $\omega$. On the other hand, if some subpopulation of NS-NS binaries has $e_{0.3 \mathrm{~Hz}} \geq 10^{-3}$, then this will be completely clear from the data itself. For these binaries, both $e_{0.3 \mathrm{~Hz}}$ and $\omega_{0.3 \mathrm{~Hz}}$ are relevant parameters, to be used both in subtraction and in projecting out residual errors. Finally, there are cases when $\omega_{0.3 \mathrm{~Hz}}$ is relevant but impossible to determine. Fortunately, even in this case, $h^{n=3}$ can simply be projected out, at very modest additional cost in bandwidth.

\section{Spin effects}

We turn now to the effects of the NS spins. Currently there are five known NS-NS binaries in our galaxy that will merge in a Hubble time (four binaries in the disk and one in globular cluster M15). In only one system-PSR J0737are the spin periods of both NSs known. For PSR J0737, $P_{\mathrm{A}}=22.7 \mathrm{~ms}$ and $P_{\mathrm{B}}=2.77 \mathrm{~s}$. In the other four systems, the radio-emitting neutron star is also a fast rotator, with $P$ ranging from $28.5 \mathrm{~ms}$ to $59.3 \mathrm{~ms}$. The fast rotators all have low spin-down rates and so appear to be recycled pulsars. From evolutionary considerations, one expects exactly one of the companions to be rapidly rotating (consistent with what we find for PSR J0737). We estimate the effect of the bodies' spins on the gravitational waveform, for this presumed-typical case where one NS is rotating relatively rapidly $(P \sim 30 \mathrm{~ms})$, while the other is slowly rotating $(P \gtrsim 1 \mathrm{~s})$.

\section{Precession of orbital plane}

If the NSs are spinning, then the orbital angular momentum vector $\vec{L}$ does not have fixed direction, but instead precesses around the binary's total angular momentum vector $\vec{J}$, due to an effective $\vec{L} \times \vec{S}$ coupling. When either (1) the two masses are nearly equal, or (2) the spin of one NS is much greater than the other, then the lowest-order precessional dynamics take an especially simple formso-called "simple precession" [26]. In fact, we expect both these conditions to be satisfied in most NS-NS binaries, since (as mentioned above), we expect only one to be rapidly rotating, and since in those binaries where both NS masses are accurately known, the masses are indeed nearly equal. Therefore we shall use the simple-precession approximation to estimate the magnitude of precessional effects on the waveform.

Following Apostolatos et al. [26], let $\lambda_{\mathrm{L}}$ be the precession amplitude; i.e., the angle between $\vec{J}$ and $\vec{L}$. While $\lambda_{\mathrm{L}}$ depends on the magnitude and direction of the spins, the precession period depends on neither (to a very good approximation). The total number of precessions, from the moment the GW frequency sweeps through $f$ until merger, is (for $M_{1} \approx M_{2}$ ):

$$
N_{\text {prec }} \approx 2.3 \times 10^{3}\left(\frac{2.8 M_{\odot}}{M(1+z)}\right)\left(\frac{0.3 \mathrm{~Hz}}{f}\right) .
$$

It is useful to define dimensionless spin parameters $\chi_{i}$ by $\chi_{i} \equiv\left|\vec{S}_{i}\right| / M_{i}^{2}$. The $\chi_{i}$ are related to the spin periods $P_{i}$ by

$$
\chi_{i}=0.036\left(\frac{I_{i}}{10^{45} \mathrm{gcm}^{2}}\right)\left(\frac{1.4 M_{\odot}}{M_{i}}\right)\left(\frac{10 \mathrm{msec}}{P_{i}}\right) .
$$

where the $I_{i}$ are the NS moments of inertia. Label the faster-rotating NS "1." Assuming $\chi_{1} \gg \chi_{2}$, the precession amplitude is simply

$$
\begin{aligned}
\lambda_{\mathrm{L}} \approx & 2.3 \times 10^{-4}\left(1-\cos ^{2} \theta_{\mathrm{LS}}\right)^{1 / 2} \\
& \cdot\left(\frac{\chi_{1}}{0.01}\right)\left(\frac{M(1+z)}{2.8 M_{\odot}}\right)\left(\frac{f}{0.3 \mathrm{~Hz}}\right)^{1 / 3} .
\end{aligned}
$$

where $\theta_{\mathrm{LS}}$ is the angle between $\vec{L}$ and $\vec{S}_{1}$. If we ignored spin-orbit precession when subtracting out the NS inspiral waveforms, we would make relative errors $\delta h / h \sim \lambda_{\mathrm{L}}$. This is $\lesssim 10^{-3}$ for $P_{1} \gtrsim 10 \mathrm{~ms}$, and so these errors would typically be benign. In any cases where $P_{1}$ is significantly less than $10 \mathrm{~ms}$, this will generally be clear from the data (from its influence on the orbital phase evolution) and these very-high-spin systems would presumably be treated as a "special class," requiring more parameters to fit them than typically necessary.

\section{Effect of spin-orbit and spin-spin terms on waveform phase}

We next consider the effect of the spin-orbit and spinspin interactions on the waveform phase. Since we have considered the effects of orbital eccentricity and orbitalplane precession in previous subsections, we simplify the analysis here by assuming that the orbit is circular and that the orbital angular momentum vector $\vec{L}$ and the two spin vectors, $\vec{S}_{1}$ and $\vec{S}_{2}$, are all aligned. Then in a postNewtonian expansion of the waveform phase $\Psi(f)$, the lowest-order terms involving the spin-orbit and spin-spin interaction are [27]

$\Psi_{\beta}(f)+\Psi_{\sigma}(f)=\frac{3}{4}(8 \pi \mathcal{M}(1+z) f)^{-5 / 3}\left[4 \beta y^{3 / 2}-10 \sigma y^{2}\right]$. 
where the terms $\beta$ and $\gamma$ are explicitly given by

$$
\begin{aligned}
\beta \equiv & \left(\frac{113}{12}+\frac{25}{4} \frac{M_{2}}{M_{1}}\right)\left(M_{1} / M\right)^{2}\left(\hat{L} \cdot \hat{S}_{1}\right) \chi_{1} \\
& +\left(\frac{113}{12}+\frac{25}{4} \frac{M_{1}}{M_{2}}\right)\left(M_{2} / M\right)^{2}\left(\hat{L} \cdot \hat{S}_{2}\right) \chi_{2}
\end{aligned}
$$

and

$$
\sigma \equiv \frac{\mu}{M} \chi_{1} \chi_{2}\left(\frac{247}{192} \hat{S}_{1} \cdot \hat{S}_{2}-\frac{721}{192}\left(\hat{L} \cdot \hat{S}_{1}\right)\left(\hat{L} \cdot \hat{S}_{2}\right)\right) .
$$

Assuming $P_{1} \sim 30 \mathrm{~ms}$ and $P_{2} \sim 1 \mathrm{~s}$, this implies $\chi_{1} \sim$ 0.01 and $\chi_{2} \sim 4 \times 10^{-4}$, and then $\beta \sim 0.04$, while $|\sigma| \sim$ $2.5 \times 10^{-6}$. So plugging in fiducial values (with $M_{1}=$ $\left.M_{2}=1.4 M_{\odot}\right)$, the spin-related phase terms are

$$
\begin{aligned}
& \Psi_{\beta}(f) \sim 6.8 \times 10^{1}\left(\frac{\beta}{0.1}\right)\left(\frac{f}{0.3 \mathrm{~Hz}}\right)^{-2 / 3}(1+z)^{-2 / 3}, \\
& \Psi_{\sigma}(f) \sim-4 \times 10^{-4}\left(\frac{\sigma}{10^{-5}}\right)\left(\frac{f}{0.3 \mathrm{~Hz}}\right)^{-1 / 3}(1+z)^{-1 / 3} .
\end{aligned}
$$

In summary, the spin-orbit term $\beta$ is clearly relevant, while spin-spin term $\sigma$ is negligible for typical cases. Thus, while it takes 6 parameters to describe (initial conditions for) the two spin vectors $\vec{S}_{1}$ and $\vec{S}_{2}$, for typical cases the spins' influence on the waveform can be adequately subsumed into a single parameter, $\beta$.

\section{High-order post-Newtonian effects, neglecting spin}

To-date, the post-Newtonian equations governing the inspiral of (quasi-)circular-orbit binaries have been derived through $\mathrm{P}^{3.5} \mathrm{~N}$ order beyond the lowest order, quadrupoleformula level [28]. Is that good enough for accurately subtracting out the merger waveforms from the BBO data, or are even higher-order treatments called for? In this subsection, we do a rough estimate that suggests that the $\mathrm{P}^{3.5} \mathrm{~N}$ equations are sufficiently accurate for this purpose (or are at least very close). Since we have considered the effects of spin and orbital eccentricity in previous subsections, for this subsection we will specialize to the case of nonspinning NSs in (quasi-)circular orbits.

We return again to the stationary-phase approximation of the waveform

$$
\tilde{h}(f) \propto(\mathcal{M}(1+z))^{5 / 6} f^{-7 / 6}[1+\ldots] \mathrm{e}^{\mathrm{i} \Psi(f)}
$$

and to the PN expansion of the phase $\Psi(f)$ :

$$
\begin{aligned}
\Psi(f)= & \text { const. }+2 \pi f t_{\mathrm{c}}+\frac{3}{4}(8 \pi \mathcal{M}(1+z) f)^{-5 / 3}\left[1+\frac{20}{9}\right. \\
& \left.\times\left(\frac{743}{336}+\frac{11 \mu}{4 M}\right) y-16 \pi y^{3 / 2}+\ldots\right] .
\end{aligned}
$$

Terms up through $\mathrm{P}^{3.5} \mathrm{~N}$ have already been calculated. We want to estimate the size of the $\mathrm{P}^{4} \mathrm{~N}$ term in the series, which corresponds to a term of the form $\frac{3}{4}(8 \pi \mathcal{M}(1+$ z) $f)^{-5 / 3} \times\left[\left(C+D(\mu / M)+E(\mu / M)^{2}+\cdots\right) y^{4}\right], \quad$ for some coefficients $C, D, E, \cdots$. The coefficient $C$ could be derived from the results in [29]; we have not done that calculation, but it is clear from [29] that $C$ is of order $10^{2}$. It seems reasonable to assume that the sum $C+D(\mu / M)+$ $E(\mu / M)^{2}+\cdots$ is also $\sim 10^{2}$. The rest of the $\mathrm{P}^{4} \mathrm{~N}$ term, $\frac{3}{4}(8 \pi \mathcal{M}(1+z) f)^{-5 / 3} y^{4}$, has magnitude

$$
4.06 \times 10^{-6}\left(\frac{M(1+z)}{2.8 M_{\odot}}\right)\left(\frac{f}{1 \mathrm{~Hz}}\right)
$$

and so the full term is of order $10^{-3}$ at $f=1 \mathrm{~Hz}$.

Thus the $\mathrm{P}^{4} \mathrm{~N}$ contribution is just at the border of being relevant. We suspect the full $\mathrm{P}^{4} \mathrm{~N}$ term will have been calculated long before BBO flies, but even today one could generate a "poor man's" $\mathrm{P}^{4} \mathrm{~N}$ waveform by simply omitting the terms involving $D(\mu / M), E(\mu / M)^{2}$, etc., but including the term $\propto C$, which we repeat is easily derivable from published results. Because $\mu / M \approx 1 / 4$, the omitted terms could easily be an order-of-magnitude smaller than the $C$-term, and so would be truly negligible.

Therefore we believe that already, today, one could produce PN waveforms that are sufficiently accurate for $\mathrm{BBO}$, or that are at least quite close. However we add that if this view turned out to be too optimistic - if it did prove difficult to generate sufficiently accurate waveforms, corresponding to realistic solutions of Einstein's equationthen there is also an obvious fallback strategy: use an enlarged space of "phenomenological waveforms," such as those developed by Buonanno et al. [30], to identify and subtract out the inspirals. The family of phenomenological waveforms would depend on a few more parameters than the physical waveforms, so projecting out subtraction errors would cost somewhat more bandwidth, but the estimates in Sec. IV B show that this cost would still likely be minimal. Therefore as long as some member of the phenomenological family lies quite close to each true waveform, meaning $\delta h / h \lesssim 10^{-3}$, the phenomenological family would suffice for the purposes of inspiral-waveform subtraction.

\section{THE DETECTION THRESHOLD $\rho_{\text {th }}$}

The GW strength (at the Earth) of any NS-NS binary is characterized by its signal-to-noise-squared, $\rho^{2}$. By $\rho^{2}$, we mean the matched-filtering $\mathrm{SNR}^{2}$ for the entire 4constellation BBO network (whose output is 12 independent GW data streams, 8 of which have good sensitivity to NS binaries). We want to estimate the threshold value $\rho_{\text {th }}^{2}$ required for the signal to be detectable. There are basically two sorts of considerations here. If one possessed infinite computing power, then this threshold value would be set just by the requirement that one has sufficient confidence in the detection (i.e., that the false-alarm rate be sufficiently low). However in practice we expect the search sensitivity to be (severely) computationally limited, which implies a somewhat higher detection threshold. 


\section{A. Lower bound on $\rho_{\text {th }}$ set by the number of effectively independent inspiral templates}

Let $N_{\mathrm{t}}$ be the number of independent templates required to cover the parameter space of NS-NS inspiral waveforms ('independent' in the sense that they have only modest overlap with each other). Then for a given threshold value $\rho_{\text {th }}$, the number of false alarms generated by this entire set is $\sim N_{\mathrm{t}} \operatorname{erfc}\left(\rho_{\mathrm{th}} / \sqrt{2}\right) \approx N_{\mathrm{t}}(2 \pi)^{-1 / 2}\left(\rho_{\mathrm{th}}\right)^{-1} \mathrm{e}^{-\rho_{\mathrm{th}}^{2} / 2}$. In practice, one would probably want this false-alarm rate to be no greater than $\sim 0.01$. How large is $N_{\mathrm{t}}$ for our problem? This has not yet been calculated, but because $\rho_{\text {th }}$ depends only logarithmically on $N_{\mathrm{t}}$, a very rough estimate will suffice for our purposes.

Consider the parameter space of "typical" inspiral waveforms, normalized by $\langle\mathbf{h} \mid \mathbf{h}\rangle=1$. These are effectively described by 10 parameters:

$$
\begin{aligned}
\lambda^{\alpha} & \equiv\left(\lambda^{1}, \ldots, \lambda^{\mathrm{N}}\right) \\
& =\left[t_{0}, \ln \mathcal{M}_{\text {eff }}, \ln \mu_{\text {eff }}, \beta, e_{0}^{2}, \Phi_{0}, \theta, \phi, \theta_{\mathrm{L}}, \phi_{\mathrm{L}}\right] .
\end{aligned}
$$

Here, $t_{0}$ is the instant of time when the ( $n=2$ piece of the) GW frequency sweeps through some fiducial value $f_{0}$ (e.g., $\left.f_{0}=0.3 \mathrm{~Hz}\right) ; \mathcal{M}_{\text {eff }} \equiv \mathcal{M}(1+z) \mu_{\text {eff }} \equiv \mu(1+z)$; $\beta$ is the spin parameter defined in Eq. (82) (and approximated here as a constant); $e_{0}^{2}$ is the square of the orbital eccentricity at $t_{0} ; \Phi_{0}$ describes the orbital phase (the angle between the orbital separation vector $\hat{r}$ and some fixed vector in the orbital plane) at $t_{0} ;(\theta, \phi)$ give the position of the source on the sky; and $\left(\theta_{\mathrm{L}}, \phi_{\mathrm{L}}\right)$ give the orientation of the binary's total angular momentum vector $\vec{L}$ (which precesses slightly, but which we can typically approximate as constant). We have omitted from this list the perihelion angle $\omega_{0}$ and 5 of the 6 parameters characterizing the two NS spin vectors, since we estimated in Sec. VC that they typically have a negligible impact on the waveform. The luminosity distance to the source, $D_{\mathrm{L}}$, has been omitted since it affects only the waveform's overall normalization.

Now imagine covering our $\mathrm{N}$-dimensional manifold of waveforms with a hypercubic grid, such that the overlap of any waveform on the manifold with the nearest gridpoint is $\geq(1-x)$, where $x$ is a number that characterizes the fineness of our grid. The number of gridpoints $N_{\mathrm{t}}$ is then [31]

$$
N_{\mathrm{t}} \approx(N / 8 x)^{N / 2} \int \sqrt{\Gamma} \mathrm{d} \lambda^{1} \ldots \mathrm{d} \lambda^{\mathrm{N}},
$$

where $\Gamma$ is the determinant of the Fisher matrix $\Gamma_{\alpha \beta} \equiv$ $\left\langle\partial_{\alpha} \mathbf{h} \mid \partial_{\beta} \mathbf{h}\right\rangle$ (again, subject to the constraint $\langle\mathbf{h} \mid \mathbf{h}\rangle=1$ ). In our case $N=10$, and we adopt $x=0.5$ as our fiducial grid spacing, so $(N / 8 x)^{N / 2} \approx 100$. We can obtain a rough estimate of the integral $\int \sqrt{\Gamma} \mathrm{d} \lambda^{1} \ldots \mathrm{d} \lambda^{\mathrm{N}}$ from estimates of the sizes of the diagonal elements of $\Gamma$, as follows. For each parameter $\lambda_{\alpha}$, let $n_{\lambda_{\alpha}}=\delta_{\lambda_{\alpha}} \mid h^{-1} \partial h / \partial \lambda_{\alpha}$ l, where $\delta_{\lambda_{\alpha}}$ is the range of integration for the $\alpha^{\text {th }}$ parameter and $\left|h^{-1} \partial h / \partial \lambda_{\alpha}\right|$ is supposed to represent some typical or "rms" value of this quantity. Then

$$
\int \sqrt{\Gamma} \mathrm{d} \lambda^{1} \mathrm{~d} \lambda^{2} \ldots \mathrm{d} \lambda^{\mathrm{N}} \lesssim n_{\lambda_{1}} n_{\lambda_{2}} \ldots n_{\lambda_{\mathrm{N}}} .
$$

The rhs represents a rough upper limit to the integral because it ignores possible cancellations in the determinant coming from the off-diagonal terms. Based on a postNewtonian expansion of the waveform, of the form shown above in Sec. VD, we derive the following order-ofmagnitude estimates for the different factors:

$$
\begin{gathered}
n_{t_{0}} \sim 10^{8}, \quad n_{\ln \mathcal{M}} \sim 10^{8}, \quad n_{\ln \mu} \sim 10^{5}, \\
n_{\beta} \sim 10^{2}, \quad n_{e_{0}^{2}} \sim 10^{2}, \quad n_{\Phi_{0}} \sim 10^{1}, \\
n_{\Omega} \sim 10^{7}, \quad n_{\Omega_{\mathrm{J}}} \sim 10^{1},
\end{gathered}
$$

where $n_{\Omega} \equiv n_{\theta} n_{\phi}, n_{\Omega_{\mathrm{J}}} \equiv n_{\theta_{\mathrm{J}}} n_{\phi_{\mathrm{J}}}$, and where we have used $\delta \beta \sim 0.5$ and $\delta e_{0}^{2} \sim 10^{-7}$. Using the above estimates, we find $N_{\mathrm{t}} \lesssim 10^{36}$. Allowing for cancellations from offdiagonal terms, it seems reasonable to assume $N_{\mathrm{t}}$ is in the range $N_{\mathrm{t}} \sim 10^{30}-10^{36}$, implying $\rho_{\text {th }} \geq 12.5-13.5$. That is, if matched filtering reveals a NS-NS inspiral with total SNR $\gtrsim 13$, then one can be confident it is not simply a randomly generated peak.

Now, one could complain that we have undercounted $N_{\mathrm{t}}$ by restricting to the parameter space of 'typical' signals, whereas among the $10^{5}-10^{6} \mathrm{NS}$ binaries that BBO will observe, there are probably some atypical ones; e.g., binaries in which both NSs are rapidly rotating. And these must also be identified and subtracted, for BBO to do its main job. This complaint has some merit, but we do not dwell on it here, since in any case we expect that in practice $\rho_{\text {th }}$ will be set not by the false-alarm rate, but by computational limitations. We turn to these next.

\section{B. Limitations due to finite computing power}

From the estimates in the previous section, one readily concludes that straightforward matched filtering for all templates in the template bank will not be possible. The simplest implementation would require of order $\sim 10^{9} N_{\mathrm{t}}$ floating point operations (since each yearlong template has $\sim 3 \times 10^{8}$ data points, if sampled at $\sim 10 \mathrm{~Hz}$ ). A well known, FFT-based trick to efficiently search over all $t_{0}$ [32] reduces this cost by a factor $\sim n_{t_{0}} /\left[3 \ln \left(10^{9}\right)\right] \sim 10^{6}$, but would still require computation speeds of $\sim 10^{28 \pm 3}$ flops (operations per second). Extrapolation of Moore's law to the year 2025 suggests that perhaps $\sim 10^{17}$ flops will be readily available, which is 11 orders of magnitude too small for the job.

Therefore one will need to devise a suboptimal (but computationally practical) search algorithm, and live with the attendant loss in sensitivity. It is beyond the scope of this paper to design such an algorithm and evaluate its efficiency. Fortunately, though, the problem of searching for NS-NS binary signals in BBO data is closely analogous to the problem of searching for unknown GW pulsars in 
LIGO data, and the problem of devising efficient search algorithms for the latter has been studied in some detail $[33,34]$. We will estimate the threshold sensitivity of BBO NS-binary searches based on this analogy, so we digress to describe optimized LIGO searches for unknown $\mathrm{GW}$ pulsars.

By unknown GW pulsars, we mean rapidly rotating NSs whose sky location, amplitude and polarization, and gravitational-wave frequency (at any instant) and frequency derivatives are all unknown, and so must be searched over; i.e., the unknown parameters are the sky location $(\theta, \phi)$, four parameters describing the amplitude, polarization, and overall phase of the waves (these can be usefully thought of as two complex amplitudes - one for each GW polarization), and the gravitational wave frequency and frequency derivatives at any instant: $f, \dot{f}, \ddot{f}$, $\dddot{f}$, etc. The typical magnitude of frequency derivatives is assumed to be $\mathrm{d}^{n} f / \mathrm{d} t^{n} \sim f / \tau^{n}$, where $\tau$ is some characteristic timescale (basically the NS's spin down-age), but these derivatives are otherwise considered independent.

For GW pulsars, we briefly describe the most efficient schemes that have been considered to-date, which are semicoherent and hierarchical (i.e., multistage) searches; we refer to Cutler et al. [34] for more details. A "semicoherent" search is one where short data stretches (say, a few days long) are all coherently searched, using some technique akin to matched filtering, and then the resulting powers from the different stretches are summed. The method is only "semicoherent" because powers are added instead of complex amplitudes; i.e., information regarding the overall phase of the signal in different stretches is discarded. This allows one to use a much coarser grid on parameter space than would be required in a fully coherent search of the same data. The basic idea of multistage searches is as follows. In the first stage one searches, semicoherently, through some fraction of the data (say, a month's worth), and identifies promising "candidates" in parameter space. One then follows up these candidates in the second stage, using a higher resolution on parameter space (a finer grid) and more data. This generates a second, sublist of candidates, which one then investigates with even higher resolution and yet more data, and so on. The idea is to reject unpromising regions in parameter space as quickly as possible, so as not to waste valuable computer resources on them. After $N_{\mathrm{s}}$ semicoherent stages like this, any remaining candidates are verified using a final, fully coherent follow-up search in a very tiny region of parameter space. A priori, the best value for $N_{\mathrm{s}}$ is unclear; it was shown in Cutler et al. [34] that for realistic GW-pulsar searches, the gains from increasing $N_{\mathrm{s}}$ saturate at $N_{\mathrm{s}}=3$ semicoherent stages.

The GW signal from a NS binary is practically the same as the signal from a low-frequency GW pulsar (except the binary's orbital frequency changes on a much shorter timescale than the spin-period of slowly rotating NSs). In both cases, the signal is essentially monochromatic at any instant, with a frequency that is slowly time-varying. In both cases there is an unknown sky position, two unknown complex amplitudes (equivalent to $D, \theta_{\mathrm{L}}, \phi_{\mathrm{L}}$, and $\Phi_{0}$ in the NS-binary case). The optimal statistic for searching over the two complex (four real) amplitudes, in both the GW-pulsar and NS-binary cases, is the $F$-statistic, which follows a chi-squared distribution with 4 degrees of freedom $[35,36]$. (The distribution is the same no matter how many detectors are combined in the analysis; the 4 d.o.f. correspond to the 2 complex - or 4 real-unknown amplitude parameters. The fact that BBO is composed of 4 LISA-like constellations outputting 12 independent data streams does not affect this counting.) The biggest difference between the two sources is that for actual GW pulsars, the signal's intrinsic amplitude can be approximated as constant over the observation time, while in the NS-binary case, the GW amplitude grows significantly during the observation time. However we do not consider this difference as very important when comparing detection thresholds, especially because the search sensitivity is really set by the early stages, where the coherent integration times will be significantly shorter than $1 \mathrm{yr}$.

The sensitivity of the GW-pulsar search is limited by the size of the parameter space one wishes to search; e.g., for an all-sky search, the size of the parameter space is set by the maximum frequency $f_{\max }$ and the shortest spin-down age $\tau_{\min }$ that one wishes to search over. We now try to choose a search-space that makes the LIGO GW pulsar search comparable in difficulty to the BBO NS-binary search. The pulsar parameters $(\dot{f}, \ddot{f}, \ddot{f})$ are closely analogous to the NS-binary parameters $(\mathcal{M}, \mu, \beta)$, which control the inspiral rate. Assuming a search up to frequency $f_{\max }=1000 \mathrm{~Hz}$, and an observation time of $T_{0}=1 \mathrm{yr}$, we estimate $n_{\dot{f}} n_{\ddot{f}} n_{\ddot{f}} \sim\left(f_{\max } T_{0}\right)^{3}\left(T_{0} / \tau_{\min }\right)^{6} \sim$ $3 \times 10^{31}\left(1 \mathrm{yr} / \tau_{\min }\right)^{6}$. Using the estimates from Eq. (82), we find that $n_{\dot{f}} n_{\ddot{f}} n_{\ddot{f}} \sim n_{\ln \mathcal{M}} n_{\ln \mu} n_{\beta}$ for $\tau_{\min } \sim 300 \mathrm{yr}$.

Continuing our comparison of the LIGO/pulsar and $\mathrm{BBO} /$ binary searches, we note that because three of BBO's four constellations have separations of order $1 \mathrm{AU}$ $(\approx 500 \mathrm{~s})$, the number of distinct patches on the sky that must be searched over is $\sim(4 \pi)(2 \pi \times 0.3 \mathrm{~Hz} \times 500 \mathrm{~s})^{2} \sim$ $10^{7}$. In comparison, for GW-pulsar searches, the number of distinct sky patches is set by the Earth's rotation about its axis, and is $\sim 3 \times 10^{4}$, or roughly 300 times fewer. (This counting assumes that the larger, but more slowly varying, Doppler shift due to Earth's motion around the Sun can be absorbed into the unknown pulsar spin-down parameters, which should be true for integration times shorter than a few months. This is good enough for our purposes, since the sensitivity of the search is really set at early stages, where only a month or two of data is examined.) On the other hand, assuming sampling at $\sim 10 \mathrm{~Hz}$ for $\mathrm{BBO}$ and sampling at $\sim 3 \mathrm{kHz}$ for the LIGO network, a yearlong GW-pulsar template contains $\sim 300$ times as many points 
as a yearlong BBO NS-binary template, so each coherent integration requires about 300 times more floating point operations in the LIGO/pulsar case than in the $\mathrm{BBO} /$ binary case.

Therefore we conclude that a LIGO/pulsar search for unknown NSs, over a parameter range set by $\left(f_{\max }=\right.$ $1000 \mathrm{~Hz}, \tau_{\min }=300 \mathrm{yr}$ ), is comparable in difficulty, computationally, to the $\mathrm{BBO} /$ binary search. The code used by Cutler et al. [34] to calculate the efficiencies of multistage GW-pulsar searches was rerun for this parameter range, assuming an available computational power of $10^{17}$ flops (and computation time of $1 \mathrm{yr}$ ). For this parameter range and computational power, LIGO/pulsar search with 3 semicoherent stages (plus a final, coherent follow-up) should be able to detect GW pulsars with $\rho$ as small as $20^{2}$ (with false-dismissal rate $=10 \%$ and false-alarm rate $=1 \%$ ). Therefore we estimate that BBO will also be able to detect and remove NS binaries with $\rho>\rho_{\text {th }}=20$ (or roughly $50 \%$ higher than the minimum $\rho_{\text {th }} \sim 13$ required for detection confidence).

However: as in the last subsection, one could complain that we have counted only the cost of searching for "typical" binaries, whereas in practice most of the computational budget may be spent on searching for the few atypical ones. Also, we have assumed (reasonably, we think, but without justification) that the computational cost of identifying all the individual sources is greater than (or at least comparable to) the cost of finding the combined best fit. Also, the comparison was made for a single false-dismissal rate (10\%), whereas we imagine that, in actual practice for the $\mathrm{BBO}$ analysis, one would want to do the BBO analysis in stages, with an ever-decreasing FD rate. Also, actual $\mathrm{BBO}$ searches may be plagued by many more outliers than would be present for the purely Gaussian noise that our sensitivity estimates were based on, and this would increase the threshold. For all these reasons, and because our method of estimating $\rho_{\text {th }} \approx 20$ "by analogy" was so crude in the first place, we will investigate the efficacy of NS-binary subtraction for a range of detection thresholds: $\rho_{\text {th }}=20,30$, or 40 .

\section{EQUATIONS CHARACTERIZING A SELF-CONSISTENT SUBTRACTION SCHEME}

Fix the values of the merger rate $\dot{n}_{0}$ (which sets the overall magnitude of $S_{\mathrm{h}}^{\mathrm{NSm}}$ ) and the detection threshold $\rho_{\text {th }}^{2}$. We want to calculate what fraction $F^{2}$ of the spectral density of the NS-binary foreground cannot be subtracted. For simplicity, we will assume that all NSs have mass $1.4 M_{\odot}$ Then our method for self-consistently determining $F^{2}$ proceeds by the following steps.

Step 1: Adopt some initial "guess" value $F_{\mathrm{G}}^{2}$. Based on this guess, we obtain a corresponding guess for the total

\footnotetext{
${ }^{2}$ I. Gholami, private communication.
}

noise level:

$$
S_{\mathrm{h}}^{\mathrm{tot}}\left(F_{\mathrm{G}}, f\right)=S_{\mathrm{h}}^{\mathrm{inst}}(f)+F_{\mathrm{G}}^{2} \cdot S_{\mathrm{h}}^{\mathrm{NSm}}(f) .
$$

Step 2: Based on this total noise level, we determine the redshift $\bar{z}(\mu)$, out to which a NS-binary with orientation $\mu \equiv \hat{L} \cdot \hat{N}$ can be detected. This boundary $\bar{z}(\mu)$ (separating detectable and undetectable sources) is determined by the equation (derived in the appendix):

$$
\rho_{\mathrm{th}}^{2}=8 \cdot \frac{2 f(\mu)}{3 \pi^{4 / 3}} \frac{(\mathcal{M}(1+\bar{z}))^{5 / 3}}{D_{L}^{2}(\bar{z})} \int_{0}^{\infty} \mathrm{d} f \frac{f^{-7 / 3}}{S_{\mathrm{h}}^{\mathrm{tot}}\left(F_{\mathrm{G}}, f\right)} .
$$

where the function $f(\mu)$ gives the dependence of the squared waveform amplitude on $\mu$ :

$$
f(\mu) \equiv \frac{\left(1+\mu^{2}\right)^{2}+4 \mu^{2}}{\int_{0}^{1} \mathrm{~d} \mu\left[\left(1+\mu^{2}\right)^{2}+4 \mu^{2}\right]}=\frac{5}{16}\left(\mu^{4}+6 \mu^{2}+1\right) .
$$

Note we have normalized $f(\mu)$ so that $\int_{0}^{1} f(\mu) d \mu=1$.

Step 4: We compute the fraction $F^{2}$ of the NS-binary foreground that is due to sources more distant than $\bar{z}(\mu)$. Based on Eqs. (10) and (12) in Phinney [1], this fraction is easily seen to be

$$
F^{2}=\frac{S_{\mathrm{h}}^{\mathrm{NSm},>\bar{z}}(f)}{S_{\mathrm{h}}^{\mathrm{NSm}}}=\frac{1}{C(0)} \int_{0}^{1} \mathrm{~d} \mu f(\mu) C(\bar{z}(\mu)),
$$

in terms of the integral

$$
C(\bar{z}) \equiv \int_{\bar{z}}^{\infty} \mathrm{d} z \frac{r(z)}{(1+z)^{4 / 3} H(z)} .
$$

where $H(z)$ and $r(z) \equiv \dot{n}(z) / \dot{n}_{0}$ are given explicitly in Eqs. (3) and (8), respectively.

So far, we have given an algorithm for computing $F\left(F_{\mathrm{G}}\right)$, i.e., for iteratively improving our initial guess $F_{\mathrm{G}}$. An initial guess $F_{\mathrm{G}}$ leads to a self-consistent solution if $F\left(F_{\mathrm{G}}\right)=F_{\mathrm{G}}$. Clearly, we can shortcut the iterative procedure simply by looking for fixed points of this function; i.e., our last step is

Step 5: Plot $F\left(F_{\mathrm{G}}\right)$, and look for fixed points, i.e., values $F_{\mathrm{G}}$ such that $F\left(F_{\mathrm{G}}\right)-F_{\mathrm{G}}=0$.

Our results are displayed in the next section.

\section{RESULTS}

As motivated in previous sections, we calculate the efficacy of foreground subtraction for 3 different values of the presentday merger rate density, $\dot{n}_{0}=$ $\left\{10^{-8}, 10^{-7}, 10^{-6}\right\} \mathrm{yr}^{-1} \mathrm{Mpc}^{-3}$, and 3 values of the detection threshold, $\rho_{\text {th }}=\{20,30,40\}$. This yields 9 different results for the self-consistent $F$ representing the fraction of the foreground noise amplitude due to undetectable (and hence unsubtractable) NS binaries. We calculate these results both for the "standard-BBO" design sensitivity, $S_{\mathrm{h}}^{\text {st.inst }}(f)$, shown in Fig. 2, and for a less sensitive version having $S_{\mathrm{h}}^{\text {inst }}=4 \times S_{\mathrm{h}}^{\text {st.inst }}(f)$, i.e., with $2 \times$ higher instru- 
mental noise amplitude. As a shorthand, we will refer to the latter as "standard/2" sensitivity. Our main results are presented in Sec. VIII A. In Sec. VIII B we gain insight into our results by exploring which binaries (i.e., which $z$ and $\mu$ ) are undetectable, for different $\dot{n}_{0}$ and $\rho_{\text {th }}$. Finally, in VIII C, we consider the case of a larger foreground, $\dot{n}_{0}=$ $10^{-5} \mathrm{yr}^{-1} \mathrm{Mpc}^{-3}$; although this merger rate is unrealistically high, this case provides a rather interesting illustration of our general method.

\section{A. Efficacy of background subtraction for BBO with standard and standard/2 sensitivity}

In Sec. VII we showed that self-consistent $F$ values are fixed points of the function $F\left(F_{\mathrm{G}}\right)$, where $F_{\mathrm{G}}$ denotes a "guessed" value for this fraction. For standard-BBO sensitivity, we find that the solution $F$ is practically independent of $\dot{n}_{0}$, for realistic merger rates. Specifically, we find

$$
F_{20}=F_{30}=0, \quad F_{40}=0.0015,
$$

where our notation is that $F_{20}$ is the solution $F$ for $\rho_{\text {th }}=$ 20 , assuming the standard-BBO instrumental noise level, and similarly for $F_{30}$ and $F_{40}$. Therefore standard-BBO is sensitive enough that the NS-NS foreground can be entirely (or almost entirely) subtracted, independent of the merger rate or detection threshold (for realistic values of those quantities).

Next we consider BBO with "standard/2" sensitivity. We denote by $F_{20}^{\prime}$ the self-consistent solution for $\rho_{\text {th }}=20$ and standard/2 sensitivity, and similarly for $F_{30}^{\prime}$ and $F_{40}^{\prime}$. For this case, the results do generally depend on $\dot{n}_{0}$ (unlike for standard-BBO). Our nine results for $F^{\prime}$, corresponding to the nine combinations of $\left(\dot{n}_{0}, \rho_{\text {th }}\right)$, are given in Table II. To illustrate how these results are derived, in Fig. 5 we show the function $F\left(F_{\mathrm{G}}\right)-F_{\mathrm{G}}$ for each $\dot{n}_{0}$, and for fixed $\rho_{\text {th }}=30$. The entries in the second row of Table II are just the $F_{\mathrm{G}}$ values where the three curves in Fig. 5 pass through zero.

None of the $F^{\prime}$ values is Table II is zero; which ones are sufficiently small that unsubtracted binaries would not significantly interfere with BBO's main goal? To answer this, in Table III we give the ratio $\left[S_{\mathrm{h}}^{\mathrm{NSm},>\bar{z}}(f) / S_{\mathrm{h}}^{\mathrm{GW}}(f)\right]^{1 / 2}$, evaluated at $f=1 \mathrm{~Hz}$, for each combination $\left(\dot{n}_{0}, \rho_{\text {th }}\right)$. Again, $S_{\mathrm{h}}^{\mathrm{NSm},>\bar{z}}(f) \equiv\left(F^{\prime}\right)^{2} S_{\mathrm{h}}^{\mathrm{NSm}}(f)$, while in Table III

TABLE II. Results for "standard/2" sensitivity. Table lists $F^{\prime}=\left(S_{\mathrm{h}}^{\mathrm{NSm},>\bar{z}} / S_{\mathrm{h}}^{\mathrm{NSm}}\right)^{1 / 2}$ for different combinations $\left(\dot{n}_{0}, \rho_{\mathrm{th}}\right) . F^{\prime}$ is the amplitude of confusion noise from unsubtractable NS binaries, divided by the total foreground amplitude.

\begin{tabular}{llll}
\hline \hline & & $S_{\mathrm{h}}^{\text {inst }}=4 \cdot S_{\mathrm{h}}^{\text {st. inst }}$ & \\
$\rho_{\text {th }} \dot{n}_{0}$ & $10^{-8}$ & $10^{-7}$ & $10^{-6}$ \\
\hline 20 & 0.0015 & 0.0015 & 0.0015 \\
30 & 0.071 & 0.077 & 0.11 \\
40 & 0.15 & 0.17 & 0.55 \\
\hline \hline
\end{tabular}

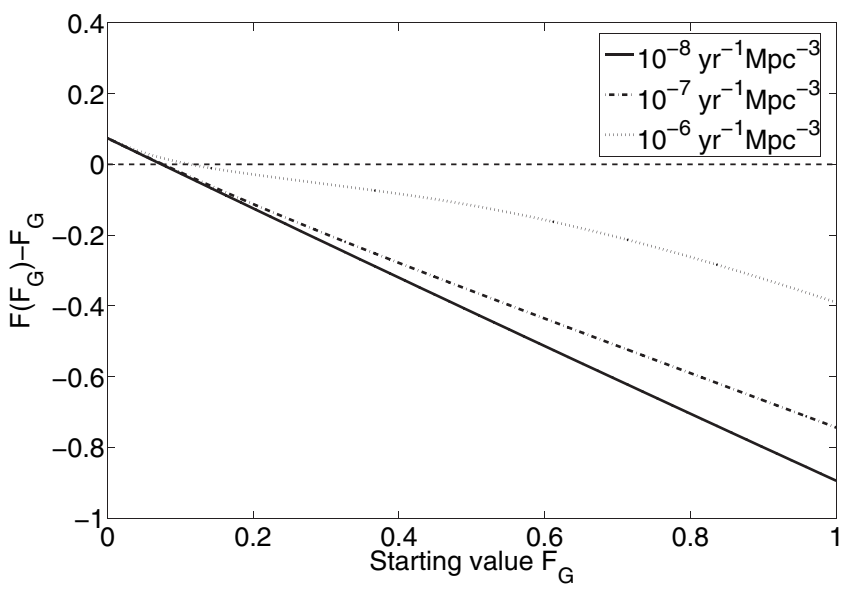

FIG. 5. Shows the function $F\left(F_{\mathrm{G}}\right)-F_{\mathrm{G}}$ for three merger rates: $\dot{n}_{0}=\left\{10^{-8}, 10^{-7}, 10^{-6}\right\} \mathrm{yr}^{-1} \mathrm{Mpc}^{-3}$. All curves are for "standard/2" sensitivity and detection threshold $\rho_{\text {th }}=30$.

$\left[S_{\mathrm{h}}^{\mathrm{GW}}(f)\right]^{1 / 2}$ is the noise spectrum for a primordial background with $\Omega_{\mathrm{GW}}(f)=10^{-16}$. Thus, ratios smaller than 1 indicate that the unsubtracted piece of the foreground is smaller than a primordial background with this energy density. We see that if $\rho_{\text {th }}=20$ (i.e., if the detection pipeline can uncover almost all NS binaries with total SNR $=20$ ), then even with standard/2 sensitivity, BBO would still be able to detect a primordial background having $\Omega_{\mathrm{GW}}(f) \geq 10^{-16}$.

However Table III also shows that if $\rho_{\text {th }}=30$ or 40 , and instrumental sensitivity is standard/2, then BBO would be unable to detect primordial background of $\Omega_{\mathrm{GW}}(f) \sim$ $10^{-16}$ (since it would be "covered up" by the unsubtractable part of the foreground).

We point out that entries for the case $\left(\dot{n}_{0}=10^{-8}, \rho_{\text {th }}=\right.$ 20) in Tables II and III should not be taken too literally, since in that case our solution $F^{\prime}$ corresponds to less than one unsubtracted binary. (A single merging NS binary at $z=5$, even with $\mu=0$, contributes $\sim 10^{-18}$ to our local $\Omega_{\mathrm{GW}}(f)$, in the BBO band.) What this means, of course, is that our solution $F^{\prime}$ lies outside the range of validity of our equations, whose derivation implicitly assumed that at least one source was undetectable. Just as clearly, our

TABLE III. Table of ratios $\left[S_{\mathrm{h}}^{\mathrm{NSm},>\bar{z}}(f) / S_{\mathrm{h}}^{\mathrm{GW}}(f)\right]^{1 / 2}$ evaluated at $f=1 \mathrm{~Hz}$, for BBO with standard/2 sensitivity. Here $S_{\mathrm{h}}^{\mathrm{GW}}(f)$ is from a primordial background with $\Omega_{\mathrm{GW}}(f)=10^{-16}$. Ratios smaller than 1 indicate that the unsubtractable part of the NSm foreground noise is smaller than this primordial background level. The results here are equivalent to those in Table II.

\begin{tabular}{|c|c|c|c|}
\hline$\rho_{\text {th }} \dot{n}_{0}$ & $\begin{array}{c}S_{\mathrm{h}}^{\text {inst }}=4 \cdot S_{\mathrm{h}}^{\text {st.inst }} \\
10^{-8}\end{array}$ & $10^{-7}$ & $10^{-6}$ \\
\hline 20 & 0.030 & 0.10 & 0.30 \\
\hline 30 & 1.4 & 4.9 & 22 \\
\hline 40 & 3.0 & 11 & 110 \\
\hline
\end{tabular}


main conclusions are unaffected. The proper interpretation of the $\left(\dot{n}_{0}=10^{-8}, \rho_{\text {th }}=20\right)$ entries is that, for these values, BBO with standard/2 sensitivity would likely detect every single NS-NS merger occurring on its past light cone.

We also repeated the above analysis for $\mathrm{BBO}$ with only standard $/ 4$ sensitivity, i.e, with $S_{\mathrm{h}}^{\text {inst }}(f)=16 \cdot S_{\mathrm{h}}^{\text {st.inst }}(f)$. This noise level is clearly inadequate, since even for $\rho_{\text {th }}=$ 20 and a low merger rate, $\dot{n}_{0}=10^{-8} \mathrm{yr}^{-1} \mathrm{Mpc}^{-3}$, we find $\left[S_{\mathrm{h}}^{\mathrm{NSm},>\bar{z}}(f) / S_{\mathrm{h}}^{\mathrm{GW}}(f)\right]^{1 / 2} \approx 3.0 \quad$ at $\quad f=1 \mathrm{~Hz}, \quad$ for $\Omega_{\mathrm{GW}}(f)=10^{-16}$.

\section{B. Further analyses of the subtraction scheme}

Here we expand on the results of the previous subsection, to improve understanding. In Fig. 6 we plot the SNR of NS binaries having $\mu=0$ (i.e., those seen edge-on: the least detectable case) as a function of $z$, under three different assumptions. The lowest curve (solid line) assumes standard BBO instrumental noise and assumes that the foreground confusion noise is the full $S_{\mathrm{h}}^{\mathrm{NSm}}(f)$ (i.e., the level before any subtraction), with $\dot{n}_{0}=$ $10^{-7} \mathrm{yr}^{-1} \mathrm{Mpc}^{-3}$. In this case, assuming $\rho_{\text {th }}=30$, all binaries out to $z \approx 1.5$ could be detected, even without first subtracting out the brightest sources. (And of course, the binaries with more favorable orientations could be detected even farther out.) In an iterative subtraction scheme, one would begin by subtracting out all the highSNR sources, which would lower the total noise and allow one to "look deeper" in succeeding iterations. For standard $\mathrm{BBO}$, this iterative scheme reaches the point where there

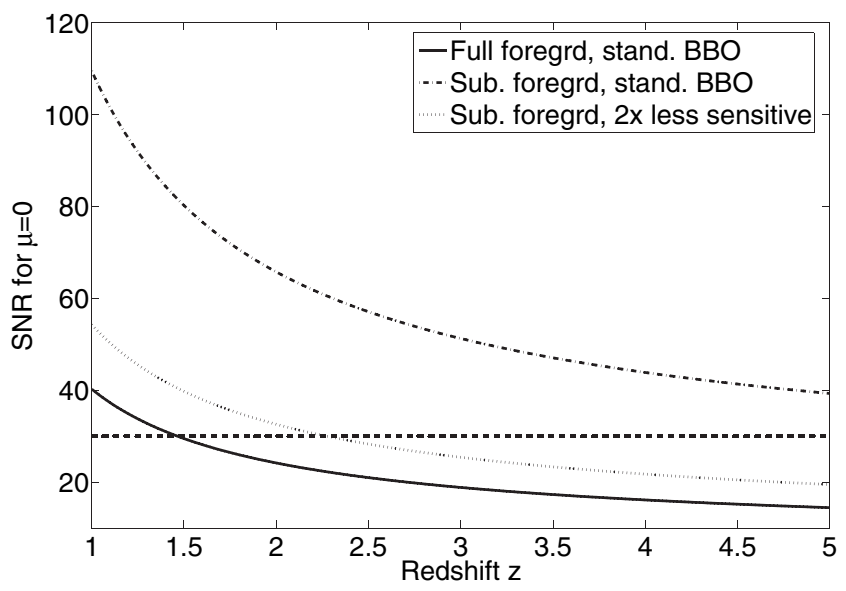

FIG. 6. Shows the SNR ratio of NS-NS mergers with $\mu=0$ in Eq. (84). The total noise level is different for each curve. The solid curve is for standard-BBO instrumental noise plus confusion noise from all NS binaries. The dotted curve is for "standard/2" instrumental noise plus foreground corresponding to $\dot{n}_{0}=10^{-7} \mathrm{yr}^{-1} \mathrm{Mpc}^{-3}$, with $F_{30}^{\prime}=0.077$ The highest curve is for standard-BBO instrumental noise and zero foreground noise. The horizontal line just highlights $\mathrm{SNR}=30$. are zero, or almost zero, unsubtracted sources, and then the total noise is just the instrumental noise.

The SNR for this "instrumental noise only" case is shown in the upper (dot-dashed) curve in Fig. 6. From this curve one sees immediately that $F=0$ is indeed a self-consistent solution: even the sources with $\mu=0$ at $z=5$ are detectable. What Fig. 6 cannot show is whether $F=0$ is the only self-consistent solution, but the rest of our analysis shows that this is true (again, for standard BBO sensitivity and $\rho_{\text {th }} \leq 30$ ). This has the practical implication that our envisioned iterative subtraction procedure should not get "stuck" at some higher $F$ value: it can keep going until all binaries have been removed. The situation for standard/2 sensitivity is different, as illustrated by the middle (dotted) curve, which corresponds to the case $\rho_{\text {th }}=30$ and $\dot{n}_{0}=10^{-7} \mathrm{yr}^{-1} \mathrm{Mpc}^{-3}$. For this case $F^{\prime}=0.077$, so the unsubtractable foreground noise is small compared to the instrumental noise, and the SNRs are roughly half the standard-BBO values. But then the $\mu=0$ binaries can only be detected to $z \approx 2.2$.

The distribution of unsubtractable binaries, for BBO with standard/2 sensitivity, is explored further in Fig. 7, which shows the maximal redshift to which NS binaries can be detected, as a function of $\mu$. The three curves are for our three detection thresholds: $\rho_{\text {th }}=20,30,40$; all assume $\dot{n}_{0}=10^{-7} \mathrm{yr}^{-1} \mathrm{Mpc}^{-3}$. For $\rho_{\text {th }}=20$ (solid curve), only a tiny corner of the $(z, \mu)$-space contains sources too weak to be detected, and the number of sources occupying that corner would be of order one (for $\dot{n}_{0}=10^{-7}$ ). For $\rho_{\text {th }}=$ 30 or 40 , the "undetectable regions" are clearly much larger, and contain several percent (or more) of all sources.

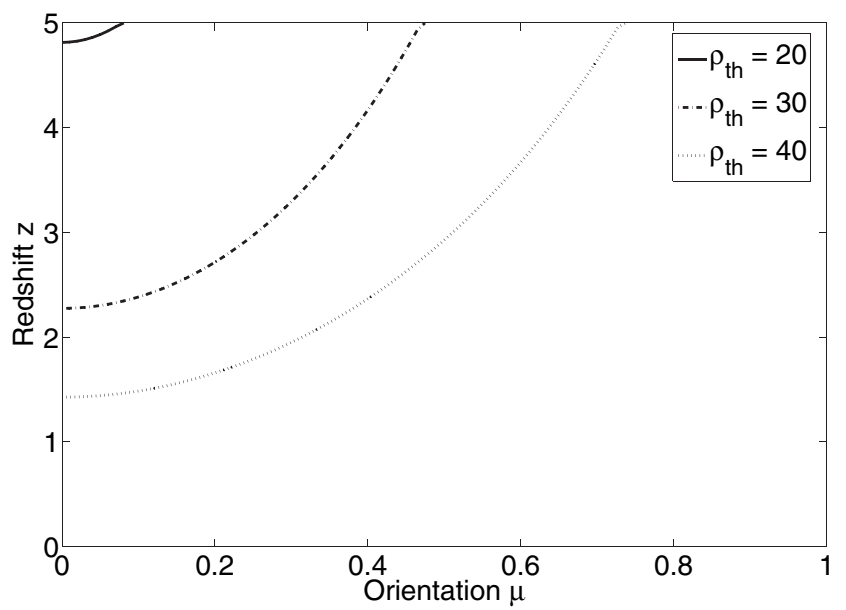

FIG. 7. Graph displays the maximum distance to which NS binaries can be detected, as function of their orientation angle $\mu \equiv \hat{L} \cdot \hat{N}$, for three different detection thresholds $\rho_{\text {th }}$. Here the instrumental sensitivity is "standard/2" and the merger rate density is $\dot{n}_{0}=10^{-7} \mathrm{yr}^{-1} \mathrm{Mpc}^{-3}$ for all three curves. 


\section{Confusion noise from a very strong NSm foreground}

The results for $F$ in Eq. (88) had basically no dependence on the merger rate $\dot{n}_{0}$, and the $F^{\prime}$ results Table II showed only weak dependence on $\dot{n}_{0}$, except at the highest values of $\dot{n}_{0}$ and $\rho_{\text {th }}$. The reason for this is simple: for BBO to succeed, the unsubtracted foreground noise must be smaller than the primordial background. Therefore, for BBO even to be "in the right ballpark," the unsubtracted foreground must be well below the instrumental noise level. In this regime, the SNR of any source is set almost entirely by $S_{\mathrm{h}}^{\text {inst }}$, and so is insensitive to $\dot{n}_{0}$. Our results are consistent with the fact that, even with sensitivity degraded by a factor 2, BBO would still be "in the ballpark" (albeit insufficient for high $\rho_{\text {th }}$ ).

However the dependence of $F$ on $\dot{n}_{0}$ becomes greater as one increases the merger rate, i.e., as unsubtractable binaries come to represent a significant fraction of the total noise. Because such cases display the full utility of our self-consistent method, we here show results for an unrealistically high merger rate: $\dot{n}_{0}=10^{-5} \mathrm{yr}^{-1} \mathrm{Mpc}^{-3}$. Figure 8 shows the function $F\left(F_{\mathrm{G}}\right)-F_{\mathrm{G}}$ for this $\dot{n}_{0}$, for standard BBO instrumental noise, and for our 3 values of $\rho_{\text {th }}$. Interestingly, each curve now has two zeroes; i.e., each case has two self-consistent solutions. A moment's thought, however, convinces one that the larger of the two solutions is the only one that is accessible by an iterative subtraction scheme. Such a scheme essentially starts at the right-most end of the curve and proceeds along it, moving to the left as sources are subtracted, until it reaches the first zero of $F\left(F_{\mathrm{G}}\right)-F_{\mathrm{G}}$. At that point, any undetected source is too deeply buried in the noise of the other undetected ones (plus the instrumental noise) to be identified. Otherwise stated: while you can selfconsistently "be at" the lower- $F_{\mathrm{G}}$ solution, the class of schemes we are considering cannot "bring you there" (and

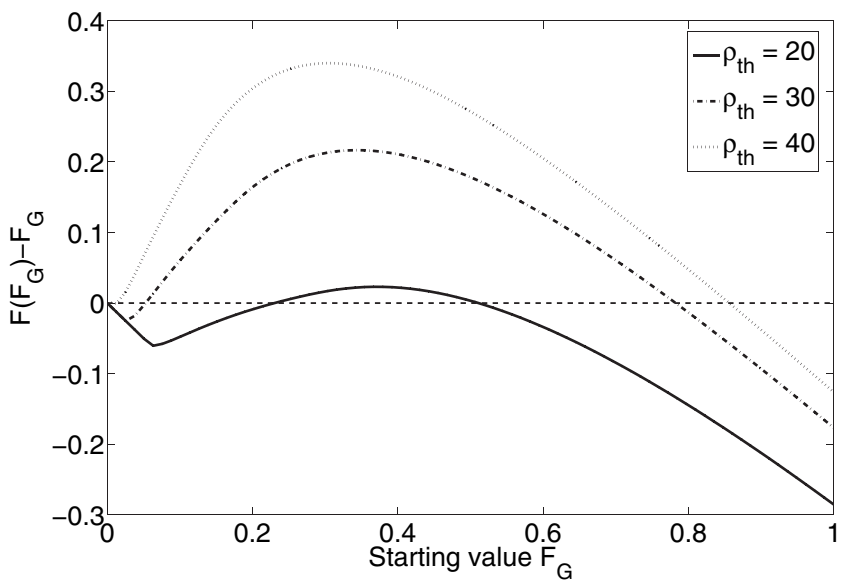

FIG. 8. Plots $F\left(F_{\mathrm{G}}\right)-F_{\mathrm{G}}$ for an unrealistically high merger rate density, $\dot{n}_{0}=10^{-5} \mathrm{yr}^{-1} \mathrm{Mpc}^{-3}$. In contrast to cases with lower $\dot{n}_{0}$, each curve now has two zeroes. However only the higher zero (larger $F$ value) can be reached by an iterative subtraction scheme. we suspect that no scheme can). Thus, we see that in a universe with $\dot{n}_{0}=10^{-5} \mathrm{yr}^{-1} \mathrm{Mpc}^{-3}$, more than half the foreground noise would be unresolvable by standard BBO.

\section{CONCLUSIONS AND FUTURE WORK}

We have calculated the efficacy of an iterative procedure for removing merging NS-binary signals from the BBO data stream, as required to detect any underlying, inflationgenerated GW background. Our calculation basically required as inputs: (a) the BBO instrumental noise curve, (b) an estimate of the extra-galactic NS-NS merger rate (as a function of $z$ ), and (c) an estimate of the inspiral SNR required for detection, with realistic computing power. We find that the current design sensitivity is sufficient to allow data analysts to subtract out the merger waveforms, for the entire range of reasonable merger rates. If $\mathrm{BBO}$ were less sensitive by a factor 2 (meaning a factor 4 higher in $S_{\mathrm{h}}^{\text {inst }}$ ), then BBO's success would depend on having a rather good detection algorithm, capable of finding almost all sources whose total SNR exceeds $\sim 20$. If BBO were less sensitive by a factor 4 , unsubtractable sources would simply "cover up" any underlying primordial background with $\Omega_{\mathrm{GW}} \lesssim$ $10^{-15}$ (or somewhat lower if NS-NS merger rates are at the low end of the predicted range).

Our goal was to estimate the efficacy of an iterative subtraction procedure, without actually trying to implement it. Of course, simulations of this procedure, to confirm our calculation or reveal holes in the argument, would also be very interesting. In particular, it would be important to confirm that our proposed projection technique on the cleaned data stream does sufficiently decontaminate it of residuals from imperfect subtractions of resolved binaries, as we have assumed in this paper. A careful simulation of the BBO data analysis process would also lead to a firmer estimate of the threshold SNR $\rho_{\text {th }}$ required for merger detection in practice, as a function of available computing power.

\section{ACKNOWLEDGMENTS}

We thank S. Phinney, N. Cornish, and N. Seto for helpful discussions regarding BBO's instrumental noise curve, and we thank I. Gholami for providing us with results on optimized pulsar search strategies, assuming $10^{17}$ Flops of computing power. Much of this work was carried out while C.C. was at the Max-Planck-Institut für Gravitationsphysik (AEI-Golm). C. C.'s work on this paper was completed at the Jet Propulsion Laboratory, California Institute of Technology, and so was also sponsored by the National Aeronautics and Space Administration. C.C. also gratefully acknowledges support from NASA Grant No. NAG5-12834. J.H. is working for the SFB407/B12 of the Deutsche Forschungsgemeinschaft and J.H. also thanks the Max-Planck-Institut für Gravitations- physik (AEI-Hannover) for its support. 


\section{APPENDIX: DERIVATION OF EQ. (84)}

In this Appendix we derive Eq. (84). We begin by averaging over all angles, including $\mu \equiv \hat{L} \cdot \hat{N}$; we return to the $\mu$-dependence near the end.

Consider first a single synthetic Michelson data stream from a single LISA-like detector. Let the waveform at the detector be $h_{i j}(t)=h_{+}(t) e_{i j}^{+}+h_{\times}(t) e_{i j}^{\times}$, where $e_{i j}^{+}$and $e_{i j}^{\times}$ are " + " and " $\times$ " polarization tensors, respectively. The average matched-filter $\mathrm{SNR}^{2}$ for some source (where the average is over source-direction and polarization angle) is given by

$$
\left\langle\mathrm{SNR}^{2}\right\rangle=4 \int_{0}^{\infty} \frac{\left|\tilde{h}_{+}(f)\right|^{2}+\left|\tilde{h}_{\times}(f)\right|^{2} \mathrm{~d} f}{S_{\mathrm{h}}(f)}
$$

where, as throughout this paper, $S_{\mathrm{h}}(f)$ is the "skyaveraged" noise spectral density. Parseval's Theorem states that

$$
\int_{0}^{\infty}\left|\tilde{h}_{+}(f)\right|^{2} \mathrm{~d} f=\frac{1}{2} \int_{-\infty}^{\infty} h_{+}^{2}(t) \mathrm{d} t,
$$

and similarly for $h_{\times}$so for a chirping signal with a slowly changing frequency $f(t)$, it is clear that

$$
\left|\tilde{h}_{+}(f)\right|^{2}+\left|\tilde{h}_{\times}(f)\right|^{2}=\frac{1}{2}\left(\bar{h}_{+}^{2}(t)+\bar{h}_{\times}^{2}(t)\right) \mathrm{d} t / \mathrm{d} f,
$$

where the overbar denotes time-averaging.

For now, consider some GW source at low redshift $(z \ll$ $1)$. Then the rate at which the source loses energy due to $\mathrm{GW}$ emission is

$$
\dot{E}(t)=4 \pi D^{2}\left(\pi f^{2} / 4\right)\left\langle\bar{h}_{+}^{2}(t)+\bar{h}_{\times}^{2}(t)\right\rangle
$$

where $D$ is its distance, and where the averaging is over all directions from the source. Therefore we have

$$
\left\langle\left|\tilde{h}_{+}(f)\right|^{2}+\left|\tilde{h}_{\times}(f)\right|^{2}\right\rangle=\frac{1}{2} \frac{\dot{E}}{\pi^{2} D^{2} f^{2}} \mathrm{~d} t / \mathrm{d} f .
$$

The product $\dot{E}(\mathrm{~d} t / \mathrm{d} f)$ equals $|\mathrm{d} E / \mathrm{d} f|$. For a circularorbit binary, the energy is approximately

$$
\begin{gathered}
E \approx-\frac{1}{2} \mu M / r \approx-\frac{1}{2} \mu(M \pi f)^{2 / 3} \\
=-\frac{1}{2} \mathcal{M}^{5 / 3}(\pi f)^{2 / 3}
\end{gathered}
$$

from which we obtain

$$
|\mathrm{d} E / \mathrm{d} f| \approx \frac{1}{3} \mathcal{M}^{5 / 3} \pi^{2 / 3} f^{-1 / 3} .
$$

Using this result along with Eqs. (A1) and (A5), we arrive at

$$
\left\langle\rho^{2}\right\rangle=\frac{2 \mathcal{M}^{5 / 3}}{3 \pi^{4 / 3} D^{2}} \int_{0}^{\infty} \frac{f^{-7 / 3} \mathrm{~d} f}{S_{\mathrm{h}}(f)} .
$$

The generalization of Eq. (A9) to arbitrary redshift is accomplished by the standard replacement [37] $\mathcal{M} \rightarrow$ $\mathcal{M}(1+z)$ and $D \rightarrow D_{\mathrm{L}}$, where $D_{\mathrm{L}}$ is the luminosity distance. The $\mu$-dependence of the waveform's strengthi.e., the $f(\mu)$ factor in Eq. (84) - follows almost immediately from, e.g., Eqs. (2a-2b) of [26]. Finally, to arrive at Eq. (84), we multiply the rhs of Eq. (A9) by a factor of 8, to account for the fact that at low-to-mid frequencies BBO is approximately equivalent to 8 independent Michelson detectors, each with the same noise density $S_{\mathrm{h}}(f)$.
[1] E. S. Phinney et al., NASA Mission Concept Study (2003).

[2] C. Ungarelli, P. Corasaniti, R. A. Mercer, and A. Vecchio, Classical Quantum Gravity 22, S955 (2005).

[3] M. S. Turner, Phys. Rev. D 55, R435 (1997).

[4] N. J. Cornish and J. Crowder, Phys. Rev. D 72, 043005 (2005).

[5] A. Buonanno, G. Sigl, G. G. Raffelt, H.-T. Janka, and E. Müller Phys. Rev. D 72, 084001 (2005).

[6] K. Danzmann et al., LISA: System and Technology Study Report, ESA-SCI(2000) 11, 2000.

[7] B. Allen, gr-qc/9604033.

[8] B. Allen and J.D. Romano, Phys. Rev. D 59, 102001 (1999).

[9] F. B. Estabrook, M. Tinto, and J. W. Armstrong, Phys. Rev. D 62, 042002 (2000).

[10] T. A. Prince, M. Tinto, S. L. Larson, and J. W. Armstrong, Phys. Rev. D 66, 122002 (2002).

[11] A. Królak, M. Tinto, and M. Vallisneri, Phys. Rev. D 70, 022003 (2004).
[12] S. L. Larson, http://www.srl.caltech.edu/shane/sensitivity/, 2005.

[13] E. S. Phinney, astro-ph/0108028.

[14] R. Schneider, V. Ferrari, S. Matarrese, and S. F. Portegies Zwart, Mon. Not. R. Astron. Soc. 324, 797 (2001).

[15] K. Belczynski, V. Kalogera, and T. Bulik, Astrophys. J. 572, 407 (2002).

[16] V. Kalogera, R. Narayan, D. N. Spergel, and J. H. Taylor, Astrophys. J. 556, 340 (2001).

[17] R. Voss and T. M. Tauris, Mon. Not. R. Astron. Soc. 342, 1169 (2003).

[18] E. S. Phinney, Astrophys. J. 380, L17 (1991).

[19] C. Cutler (to be published).

[20] C. Cutler and E.E. Flanagan, Phys. Rev. D 49, 2658 (1994).

[21] L. A. Wainstein and V. D. Zubakov, Extraction of Signals from Noise (Dover, New York, 1962).

[22] P. C. Peters, Phys. Rev. B 136, 1224 (1964).

[23] A. Królak, K. D. Kokkotas, and G. Schäfer, Phys. Rev. D 
52, 2089 (1995).

[24] P. C. Peters and J. Mathews, Phys. Rev. 131, 435 (1963).

[25] L. Wen, Astrophys. J. 598, 419 (2003).

[26] T. A. Apostolatos, C. Cutler, G. J. Sussman, and K. S. Thorne, Phys. Rev. D 49, 6274 (1994).

[27] A. Vecchio, Phys. Rev. D 70, 042001 (2004).

[28] S. Nissanke and L. Blanchet, Classical Quantum Gravity 22, 1007 (2005).

[29] M. Sasaki and H. Tagoshi, Living Rev. Relativity (2003).

[30] A. Buonanno, Y. Chen, Y. Pan, and M. Vallisneri, Phys. Rev. D 70, 104003 (2004).

[31] B. J. Owen, Phys. Rev. D 53, 6749 (1996).
[32] B.F. Schutz, The Detection of Gravitational Waves (Cambridge University Press, Cambridge, England, 1991).

[33] P. R. Brady and T. Creighton, Phys. Rev. D 61, 082001 (2000).

[34] C. Cutler, I. Gholami, and B. Krishnan (2005), Phys. Rev. D 72, 042004 (2005).

[35] S. Bose, A. Pai, and S. Dhurandhar, Int. J. Mod. Phys. D 9, 325 (2000).

[36] C. Cutler and B.F. Schutz (2005), Phys. Rev. D 72, 063006 (2005).

[37] D. Markovic, Phys. Rev. D 48, 4738 (1993). 